\title{
Spin asymmetries in quasielastic charged current neutrino-nucleon scattering
}

\author{
Krzysztof M. Graczyk $\odot^{*}$ and Beata E. Kowal $\odot^{\dagger}$ \\ Institute of Theoretical Physics, University of Wrocław, plac Maxa Borna 9, 50-204, Wrocław, Poland
}

(Received 4 December 2019; accepted 23 March 2020; published 10 April 2020)

\begin{abstract}
The work concerns the quasielastic charged current neutrino-neutron and antineutrino-proton interactions. Single, double, and triple spin asymmetries are computed and analyzed. The spin asymmetries are sensitive to the axial form factor of the nucleon. In particular, the target-recoil double spin asymmetry and the lepton-target-recoil triple spin asymmetry depend strongly on the axial form factor of the nucleon. Indeed, the sign and shape of these components depend on the axial mass parameter. All the asymmetries, except the lepton polarization, are observables well suited to study the nonstandard interactions described by the second-class current contribution.
\end{abstract}

DOI: $10.1103 /$ PhysRevD.101.073002

\section{INTRODUCTION}

The spin observables, in particles collisions, have been intensively studied for many years [1-4]. They contain information about the structure of the scattering amplitude and the structure of the particles participating in the interaction. The meson photoproduction is a good example of the process for which the spin observables are utilized to constrain the form of the scattering amplitude $[5,6]$. Another example is the elastic ep scattering. Indeed, from the measurements of the spin asymmetries, the form factor ratio $G_{E}^{p} / G_{M}^{p}$ is extracted [7-12], $G_{E / M}^{p}$ is electric/magnetic form factor of the proton.

In this work, we consider the polarization observables in the quasielastic charged current (CCQE) neutrino (antineutrino)-neutron (proton) scattering. Progress in studies of the fundamental properties of the neutrinos requires more precise knowledge of the neutrino-nucleon and neutrinonucleus cross sections [13,14]. The CCQE $\nu_{\mu}$-nucleon scattering is the process that is very important for the experimental investigations of the neutrino oscillation phenomenon. Indeed, in the long-baseline experiments, such as T2K [15] or Nova [16], the CCQE $\nu_{\mu}$-nucleus events are analyzed to obtain the neutrino oscillation parameters and $C P$-violation phase $(C P$ denotes charge conjugation parity symmetry). On the other hand, the theoretical description of the CCQE neutrino-nucleon

\footnotetext{
*krzysztof.graczyk@uwr.edu.pl

beata.kowal@uwr.edu.pl
}

Published by the American Physical Society under the terms of the Creative Commons Attribution 4.0 International license. Further distribution of this work must maintain attribution to the author(s) and the published article's title, journal citation, and DOI. Funded by SCOAP ${ }^{3}$. interaction is the input for the nuclear models for the $\nu$-nucleus scattering.

The CCQE $\nu_{\mu}$-nucleon interaction is described by a simple model. Indeed, the scattering amplitude is parametrized by four form factors, two vector and two axial. The axial contribution dominates the CCQE cross section. After some simplifications, it is parametrized by the axial form factor $F_{A}$ [17]. The accurate estimate of the axial nucleon form factor $F_{A}$ is necessary to reduce the uncertainty of the predictions of the neutrino-nucleus scattering cross sections, which is desired to lower the systematic uncertainty of the measurement of the oscillation parameters and the $C P$-violation phase, in the lepton sector.

The axial nucleon form factor is extracted from the neutrino-nucleus scattering data. However, it is a difficult task because of the complexity of the nuclear effects [18]. So far, the most accurate information about $F_{A}$ is obtained from the analysis of the neutrino-hydrogen and the neutrino-deuteron scattering data [19-25]. Usually, to simplify the analysis, it is assumed that $F_{A}$ has a dipole form. However, an effort has been made to search for deviations from the dipole shape [26-30]. Unfortunately, to get a clear evidence for a nondipole dependence, it is necessary to collect more informative data.

The so-called second-class current (SCC) [31] describes a contribution to the electroweak nucleon vertex not allowed by the Standard Model. In last several decades, SCC has been studied theoretically (for recent review, see Fatima et al. [32]) and experimentally [22,33-35]. However, no evidence for the SCC contribution has been obtained so far.

The studies of the spin properties in the CCQE $\nu$-nucleon interactions should extend our knowledge about the elementary electroweak nucleon vertex. Indeed, we showed [36-38] that the spin observables contain nontrivial 
information about the nonresonant background contribution in the single pion production (SPP) processes induced by interactions of neutrinos with nucleons. In this work, we aim to study the axial and the SCC contributions to the spin observables in the CCQE scattering. In particular, we focus on the investigation of the sensitivity of the spin observables to the axial and SCC form factors.

We consider seven spin observables. Among three single spin asymmetries: the recoil polarization asymmetry, the lepton asymmetry, and the polarized target asymmetry, the last one has been not discussed for the CCQE scattering yet. But it was studied for the SPP in the neutrino-nucleon scattering [38]. Already in the 60s, Adler [39], Pais [40], and Block [41] investigated the properties of the polarization of the recoil nucleon and the charged lepton in the CCQE neutrino-nucleon interactions. A report by Llewellyn Smith [42] summarizes these works. Recently, the polarization properties of the $\tau$-lepton, produced in the CCQE $\nu_{\tau}$-nucleon scattering, have been investigated [43-45]. Bilenky and Christova studied the axial contribution to the nucleon polarization in the CCQE $\nu_{\mu}$-nucleon scattering [46,47]. Whereas in Ref. [32], the SCC contribution to the lepton and the recoil nucleon polarizations is estimated. Eventually, the polarization properties of the final lepton and the knockout nucleon, in the CCQE neutrino-nucleus scattering, are discussed in Refs. [48-50] and [51], respectively.

Besides the polarization asymmetries, we consider the lepton-recoil, target-lepton, target-recoil double spin asymmetries, as well as target-lepton-recoil triple spin asymmetry. All these observables have been not studied for the CCQE scattering yet.

To discuss the physical properties of the asymmetries, we calculate their components in spin basis. Eventually, we provide with the numerical analysis of the results. We show that the spin observables contain the information about the axial structure of the nucleon, which is complementary to the spin averaged cross section measurements. They are also sensitive to possible signals from the nonstandard interactions, described by SCCs. In particular, the target-recoil asymmetry is sensitive to the axial form factor. Indeed, the sign and the shape of transverse-transverse and normalnormal components of the target-recoil asymmetry depend on the value of the axial mass $M_{A}$. Similar dependence is obtained for the components of the lepton-target-recoil asymmetry (longitudinal-normal-normal, longitudinaltransverse-transverse, and longitudinal-transverse-normal).

The spin asymmetries are experimentally investigated in hadron-hadron collisions (meson-nucleon, baryon-baryon), photoproduction processes, lepton-lepton, and leptonnucleon collisions [52-54]. However, in the CCQE $\nu N$ scattering, the spin asymmetries have not been measured yet. Experimental studies of the neutrino interactions are more challenging than, for instance, the investigations of the electron-nucleon interactions. On the other hand, the measurement of the spin observables demands well control of the beam and the target $[55,56]$. Moreover, to study the interactions of the neutrinos with neutrons, the neutrinonucleus scatterings are analyzed. It is known that the polarization observables are modified by the nuclear effects [48], but in this work, we focus on the simplest scenario, interactions of neutrinos with free nucleons.

Despite the difficulties described above, already in the $60 \mathrm{~s}$, Block [41] proposed the measurement of the recoil polarization of the nucleon in the CCQE $\nu N$ scattering. The idea was to adopt the bubble chamber detector technique. The development of new experimental techniques, such as the liquid argon time projection chamber [57-59], should allow performing precision measurements of the neutrino scattering cross sections. We hope that with the usage of the new experimental methods, the measurement of the spin observables, presented in this paper, will be feasible in the future.

The paper is organized as follows: in Sec. II, the necessary formalism is introduced. Section III contains the definition of the spin observables and Sec. IV presents the discussion of the numerical results. Appendix presents convention and normalization used in the paper. In the Supplemental Material [60], we give the components of the asymmetries calculated in spin basis as well as the analytic formulas for the spin asymmetries in the vector and the tensor form.

\section{FORMALISM}

\section{A. Kinematics}

Let us consider two CCQE scattering processes,

$$
\begin{aligned}
& \nu_{\mu}(k)+n(p) \rightarrow \mu^{-}\left(k^{\prime}\right)+p\left(p^{\prime}\right), \\
& \bar{\nu}_{\mu}(k)+p(p) \rightarrow \mu^{+}\left(k^{\prime}\right)+n\left(p^{\prime}\right),
\end{aligned}
$$

where $N=p$ (proton) or $N=n$ (neutron).

The momenta of neutrino, lepton, target nucleon, and recoil nucleon are denoted as it is given below.

$$
\begin{aligned}
k^{\alpha} & =(E, \mathbf{k}), \quad k^{\prime \alpha}=\left(E_{k^{\prime}}, \mathbf{k}^{\prime}\right), \quad p^{\alpha}=\left(E_{p}, \mathbf{p}\right), \\
p^{\prime \alpha} & =\left(E_{p^{\prime}}, \mathbf{p}^{\prime}\right),
\end{aligned}
$$

where $E_{k}=\sqrt{\mathbf{k}^{2}+m^{2}}$. In the next part of the text, $m$ and $M$ stand for the lepton and the averaged nucleon mass, respectively.

The four-momentum transfer is defined by

$$
q^{\alpha}=k^{\alpha}-k^{\prime \alpha}=(\omega, \mathbf{q}),
$$

where $\omega$ and $\mathbf{q}$ denote the transfer of an energy and a momentum, respectively.

The components of the asymmetry tensors are calculated in the coordinate system so that $z$-axis goes along the 


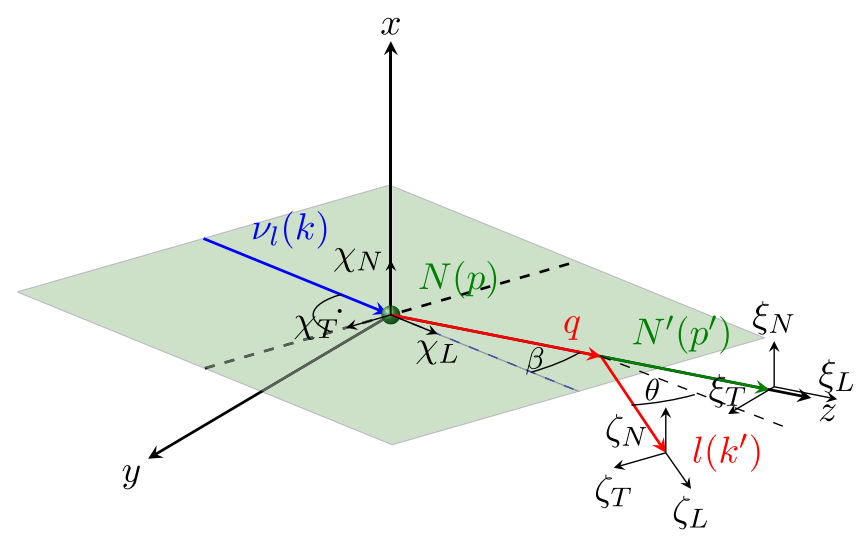

FIG. 1. The polarization basis vectors for the fermions in the CCQE $\nu$-nucleon scattering. The full (green) ball denotes the target. The blue, red, and green arrows are the momenta of the neutrino, the charged lepton, and the recoil nucleon, respectively. The vectors $\chi, \xi$, and $\zeta$ are the elements of the basis for the spins of target, recoil nucleon, and lepton, respectively.

momentum $\mathbf{q}$, hence $q^{\mu}=(\omega, 0,0,|\mathbf{q}|)$, the axis $z$ and $y$ lie in the scattering plane spanned by $\mathbf{k}^{\prime}$ and $\mathbf{k}$ vectors, whereas $x$ is normal to the scattering plane; see Fig. 1.

The spin asymmetries are expressed as functions of the Mandelstam variables,

$$
\begin{aligned}
t & =q^{\mu} q_{\mu} \equiv q \cdot q=q^{2}=-Q^{2}, \quad s=(p+k)^{2}, \\
u & =\left(p-k^{\prime}\right)^{2} .
\end{aligned}
$$

The analytic formulas take simpler form when the scalar

$$
s_{u} \equiv s-u
$$

is used.

\section{B. Cross section}

The differential crosss ection, in the laboratory frame, is given by the formula

$$
\frac{d^{6} \sigma}{d^{3} \mathbf{k}^{\prime} d^{3} \mathbf{p}^{\prime}}=\frac{|\mathcal{M}|^{2}}{64 \pi^{2} M E E_{k^{\prime}} E_{p^{\prime}}} \delta^{(4)}\left(p^{\prime}+k^{\prime}-k-p\right),
$$

where $\mathcal{M}$ is the scattering matrix. In the one-boson exchange approximation, it has the form

$$
i \mathcal{M}=\cos \theta_{C} \frac{G_{F}}{\sqrt{2}} j_{\mu}\left(\mathbf{k}^{\prime}, s^{l} ; \mathbf{k}\right) J^{\mu}\left(\mathbf{p}^{\prime}, s^{N^{\prime}} ; \mathbf{p}, s^{N}\right),
$$

where $G_{F}=1.16639 \times 10^{-5} \mathrm{GeV}^{-2}$ is a Fermi constant; $\theta_{C}$ is the Cabibbo angle, $\cos \theta_{C}=0.9737$; by $s^{l}, s^{N}$, and $s^{N^{\prime}}$ the charged lepton, the target nucleon, and the recoil nucleon spins are denoted, respectively. The spin of the neutrino is omitted as it is a state of definite helicity.
The $\mathcal{M}$ matrix is given by the contraction of lepton current,

$$
j_{\alpha}\left(\mathbf{k}^{\prime}, s^{l} ; \mathbf{k}\right)=\bar{u}\left(\mathbf{k}^{\prime}, s^{l}\right) \gamma_{\alpha}\left(1-x \gamma_{5}\right) u(\mathbf{k}),
$$

with hadron current,

$$
J^{\mu}\left(\mathbf{p}^{\prime}, s^{N^{\prime}} ; \mathbf{p}, s^{N}\right)=\bar{u}\left(\mathbf{p}^{\prime}, s^{N^{\prime}}\right) \Gamma_{+}^{\mu}(q) u\left(\mathbf{p}, s^{N}\right),
$$

where $\Gamma_{+}^{\mu}(q)$ is the electroweak nucleon vertex. Its analytic form is given in the next section.

After several simplifications, the differential cross section reads

$$
\frac{d \sigma_{0}}{d t} \equiv \frac{d \sigma}{d t}\left(s^{N^{\prime}}=s^{l}=s^{N}=0\right)=\frac{G_{F}^{2} \cos ^{2} \theta_{C}}{128 \pi M^{2} E^{2}} \mathcal{I},
$$

where

$$
\mathcal{I} \equiv \mathrm{L}_{\mu \nu}\left(s^{l} \rightarrow 0\right) \mathrm{H}^{\mu \nu}\left(s^{N} \rightarrow 0, s^{N^{\prime}} \rightarrow 0\right) .
$$

$\mathrm{H}^{\mu \nu}\left(s^{N^{\prime}}, s^{N}\right)=\operatorname{Tr}\left(\Lambda\left(p^{\prime}, s^{N^{\prime}}, M\right) \Gamma_{+}^{\mu} \Lambda\left(p, s^{N}, M\right) \gamma_{0} \Gamma^{\nu^{\dagger}} \gamma_{0}\right)$

is the hadronic tensor, whereas

$$
\mathrm{L}_{\mu \nu}=\operatorname{Tr}\left(\Lambda\left(k^{\prime}, s^{l}, m\right) \gamma_{\mu}\left(1-x \gamma_{5}\right) \not k \gamma_{\nu}\left(1-x \gamma_{5}\right)\right)
$$

is the lepton tensor, $x= \pm 1$ for neutrino/antineutrino, $\not k=\gamma_{\mu} k^{\mu}$. In the above expressions, the projection operator

$$
\Lambda(p, s, M) \equiv \frac{1}{2}(\not p+M)\left(1+\gamma_{5} \not\right)
$$

is introduced. Notice that for fully polarized state $s_{\mu} s^{\mu}=-1$. The differential cross section, averaged over initial spins and summed over final spins, reads $d \bar{\sigma} / d t=$ $4 d \sigma_{0} / d t$.

\section{Hadronic current}

The hadronic current has the vector-axial structure. Indeed, the electroweak nucleon vertex reads $[42,61]$

$$
\begin{aligned}
\Gamma_{+}^{\mu}(q)= & \gamma^{\mu} F_{1}^{V}(t)+i \sigma^{\mu \nu} q_{\nu} \frac{F_{2}^{V}(t)}{2 M}+q^{\mu} \frac{F_{3}^{V}(t)}{M} \\
& -\left(\gamma^{\mu} F_{A}(t)+q^{\mu} \frac{F_{P}(t)}{2 M}+i \sigma^{\mu \nu} q_{\nu} \frac{F_{3}^{A}(t)}{M}\right) \gamma_{5} .
\end{aligned}
$$

The functions $F_{i}^{V}$ and $F_{i}^{A}(i=1,2,3)$ refer to vector and axial form factors of the nucleon, respectively. If one assumes time reversal invariance (standard recruitment), then all form factors are real. 

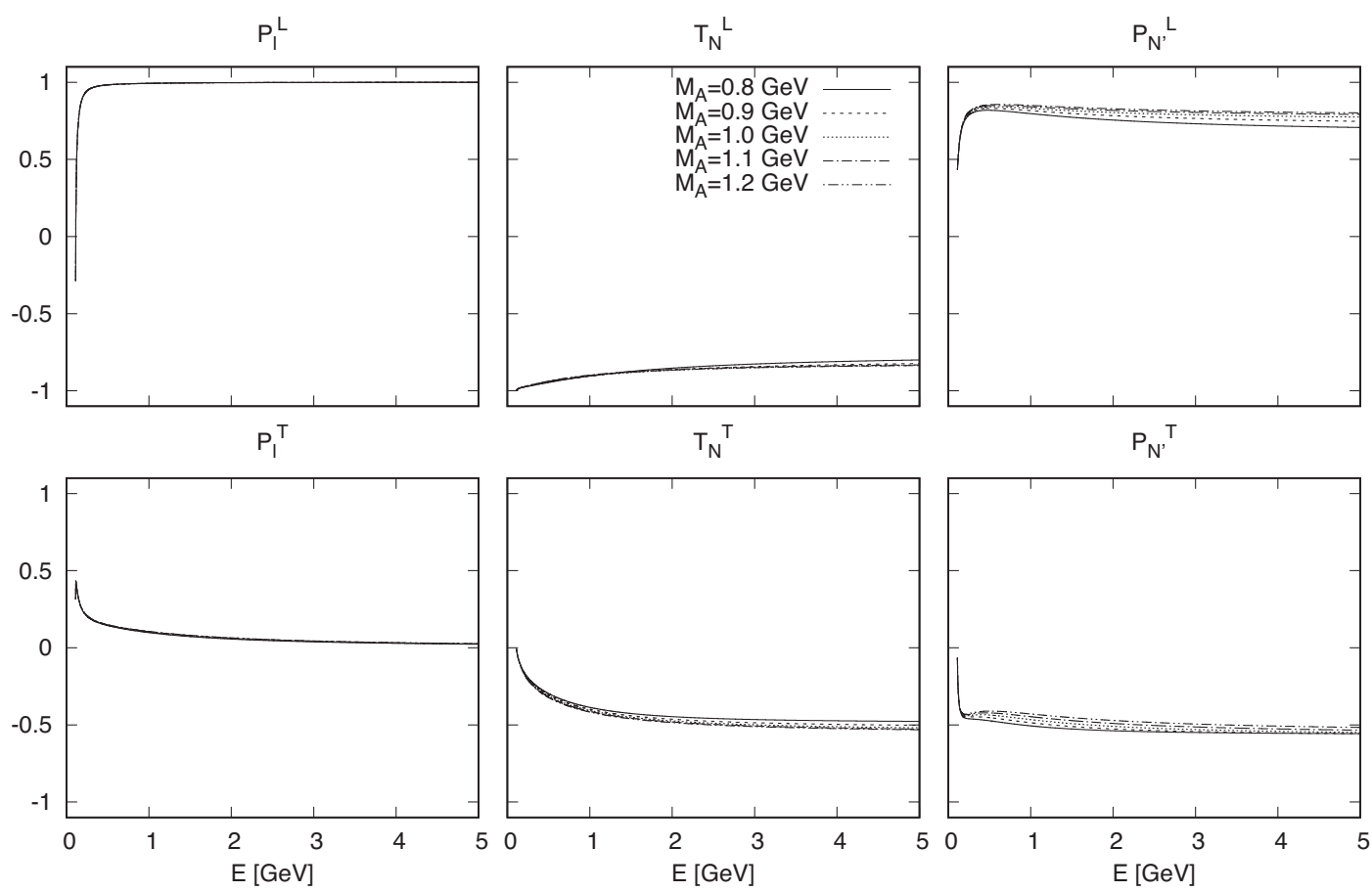

FIG. 2. Energy dependence of the components of single spin asymmetries $\mathcal{P}_{l}(E)$ (left column), $\mathcal{T}_{N}(E)$ (middle column), and $\mathcal{P}_{N^{\prime}}(E)$ (right column), given by the ratios of the total cross sections; see Eqs. (50)-(52). Results obtained for the CCQE $\nu_{\mu} n$ scattering, the axial mass $M_{A}=0.8,0.9,1.0,1.1,1.2 \mathrm{GeV}$, and $F_{3}^{A}=0$. The longitudinal/transverse components are shown in top/bottom row.

It is convenient to characterize the elements of the weak current according to the $G$-parity transformation [31]. The vector current contributions, described by $F_{1}^{V}$ and $F_{2}^{V}$ form factors, transform similarly as their analogs in the Standard Model. Similarly, the axial current induced by the $F_{A}$ and $F_{P}$ has the same $G$-parity property as their analog in the Standard Model. Hence, the standard part of the electroweak vertex reads
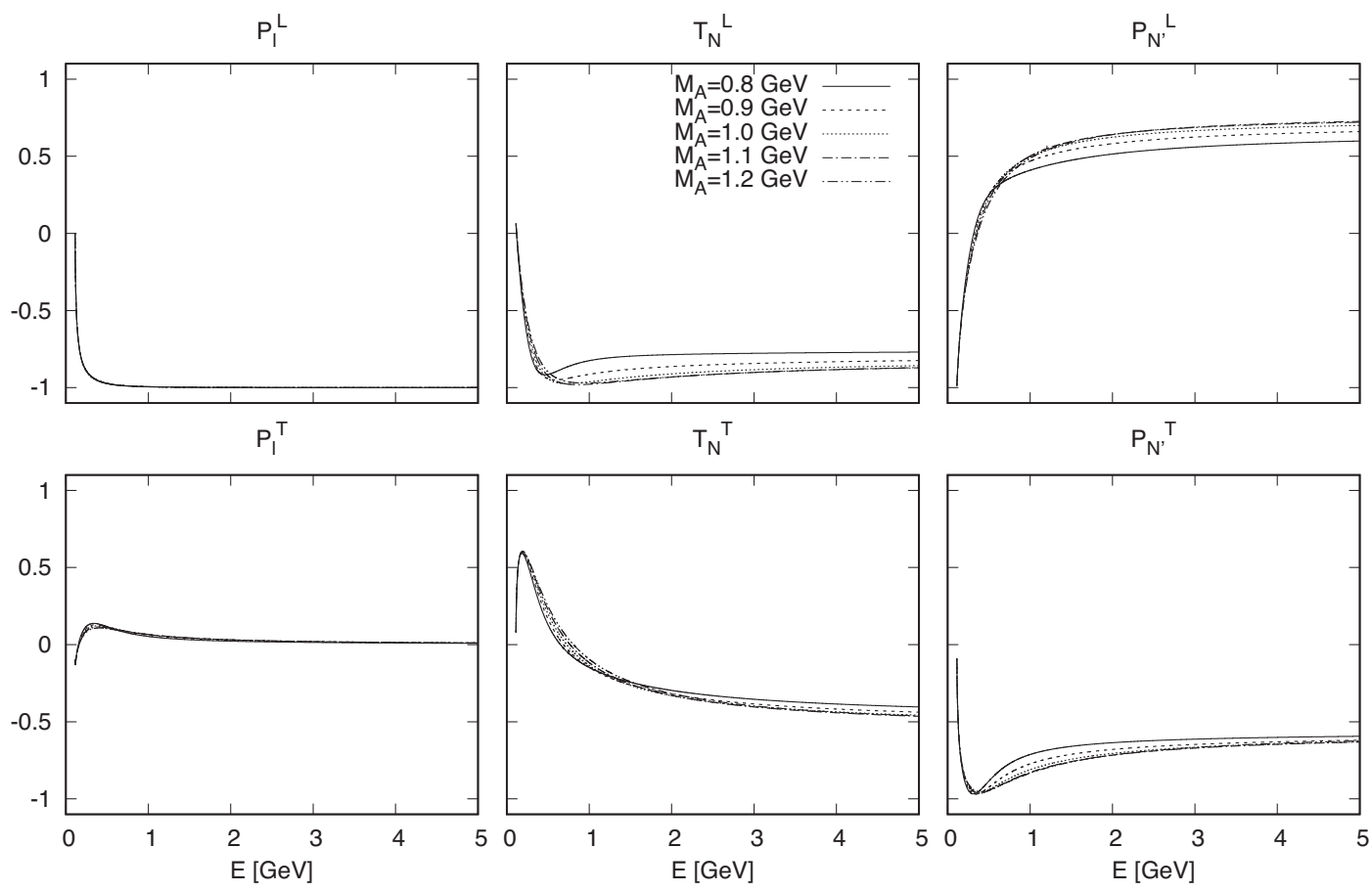

FIG. 3. Caption the same as in Fig. 2 but for the CCQE $\bar{\nu}_{\mu} p$ scattering. 

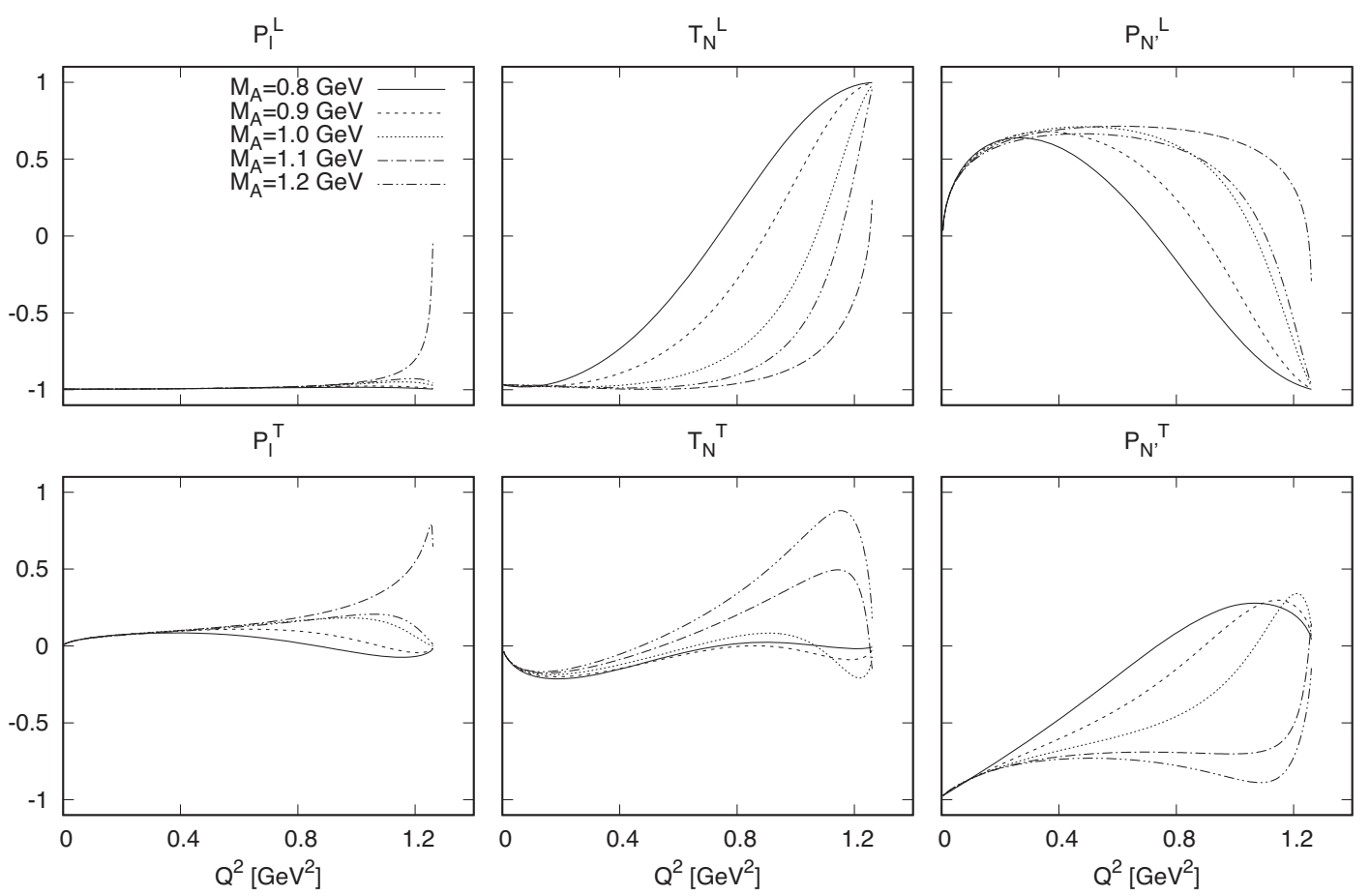

FIG. 4. Dependence of the components of $\mathcal{P}_{l}\left(E=1 \mathrm{GeV}, Q^{2}\right)$ (left column), $\mathcal{T}_{N}\left(E=1 \mathrm{GeV}, Q^{2}\right)$ (middle column), and $\mathcal{P}_{N^{\prime}}(E=$ $1 \mathrm{GeV}, Q^{2}$ ) (right column) on $Q^{2}$; see Eq. (42). Results obtained for the CCQE $\bar{\nu}_{\mu} p$ scattering, $M_{A}=0.8,0.9,1.0,1.1,1.2 \mathrm{GeV}$, $F_{3}^{A}=0$, and for energy $E=1 \mathrm{GeV}$. In the top/bottom row, longitudinal/transverse components are plotted.

$$
\begin{aligned}
\Gamma_{+(1)}^{\mu}(q) & =\gamma^{\mu} F_{1}^{V}(t)+\frac{i \sigma^{\mu \nu} q_{\nu}}{2 M} F_{2}^{V}(t) \\
& -\left(\gamma^{\mu} F_{A}(t)+\frac{q^{\mu}}{2 M} F_{P}(t)\right) \gamma_{5} .
\end{aligned}
$$

Then $\bar{u}\left(\mathbf{p}^{\prime}\right) \Gamma_{+(1)}^{\mu} u(\mathbf{p})$ is called the first-class current.

There are many phenomenological parametrizations of the vector form factors; see, e.g., [62]. But without losing the generality of the discussion, we consider the dipole parametrization [63],

$$
\begin{aligned}
& F_{1}^{V}(t)=\frac{\left(1-\left(t / 4 M^{2}\right)\left(\mu_{p}-\mu_{n}\right)\right)}{1-t / 4 M^{2}} G_{D}(t), \\
& F_{2}^{V}(t)=\frac{\mu_{p}-\mu_{n}-1}{1-t / 4 M^{2}} G_{D}(t),
\end{aligned}
$$

where $\mu_{p}=2.793$ and $\mu_{n}=-1.913$ are proton and neutron magnetic moments expressed in nuclear magneton unit, whereas

$$
G_{D}(t)=\left(1-\frac{t}{M_{V}^{2}}\right)^{-2}, \quad M_{V}=0.84 \mathrm{GeV} .
$$

The parametrization of the axial form factor, used in the analysis, is given in Sec. IV. The pseudoscalar axial form factor, $F_{P}$, is related to $F_{A}$ as follows [17]:

$$
F_{P}(t)=\frac{4 M^{2}}{\left(m_{\pi}^{2}-t\right)} F_{A}(t),
$$

where $m_{\pi}$ is the pion mass.
If the $G$ parity is weakly violated, then the form factors $F_{3}^{V}$ and $F_{3}^{A}$ do not vanish, and contribute to the so-called second-class current, described by the vertex

$$
\Gamma_{+(2)}^{\mu}(q)=q^{\mu} \frac{F_{3}^{V}(t)}{M}-i \sigma^{\mu \nu} q_{\nu} \gamma_{5} \frac{F_{3}^{A}(t)}{M} .
$$

We impose the conserved vector current theorem to constrain the SCC. Then, $F_{3}^{V}=0$; however, there is no such constraint for the axial $F_{3}^{A}$ form factor. Therefore, $F_{3}^{A}$ is the function that parametrizes the SCC contribution.

To distinguish between the first and the second-class contributions, we introduce the notation

$$
\mathrm{H}^{\mu \nu}=\mathrm{H}_{(1)}^{\mu \nu}+\mathrm{H}_{(12)}^{\mu \nu}+\mathrm{H}_{(2)}^{\mu \nu},
$$

where

$$
\begin{aligned}
\mathrm{H}_{(1)}^{\mu \nu} \equiv & \operatorname{Tr}\left(\Lambda\left(p^{\prime}, s^{N^{\prime}}, M\right) \Gamma_{+,(1)}^{\mu} \Lambda\left(p, s^{N}, M\right) \bar{\Gamma}_{+,(1)}^{\nu}\right) \\
\mathrm{H}_{(2)}^{\mu \nu} \equiv & \operatorname{Tr}\left(\Lambda\left(p^{\prime}, s^{N^{\prime}}, M\right) \Gamma_{+,(2)}^{\mu} \Lambda\left(p, s^{N}, M\right) \bar{\Gamma}_{+,(2)}^{\nu}\right) \\
\mathrm{H}_{(12)}^{\mu \nu} \equiv & \operatorname{Tr}\left(\Lambda\left(p^{\prime}, s^{N^{\prime}}, M\right) \Gamma_{+,(1)}^{\mu} \Lambda\left(p, s^{N}, M\right) \bar{\Gamma}_{+,(2)}^{\nu}\right) \\
& +\operatorname{Tr}\left(\Lambda\left(p^{\prime}, s^{N^{\prime}}, M\right) \Gamma_{+,(2)}^{\mu} \Lambda\left(p, s^{N}, M\right) \bar{\Gamma}_{+,(1)}^{\nu}\right) .
\end{aligned}
$$

In the above, $\mathrm{H}_{(1)}^{\mu \nu}$ refers to the standard hadronic tensor (the first-class contribution only), whereas $\mathrm{H}_{(12)}^{\mu \nu}$ and $\mathrm{H}_{(2)}^{\mu \nu}$ describe the second-class current corrections to the hadronic tensor. 

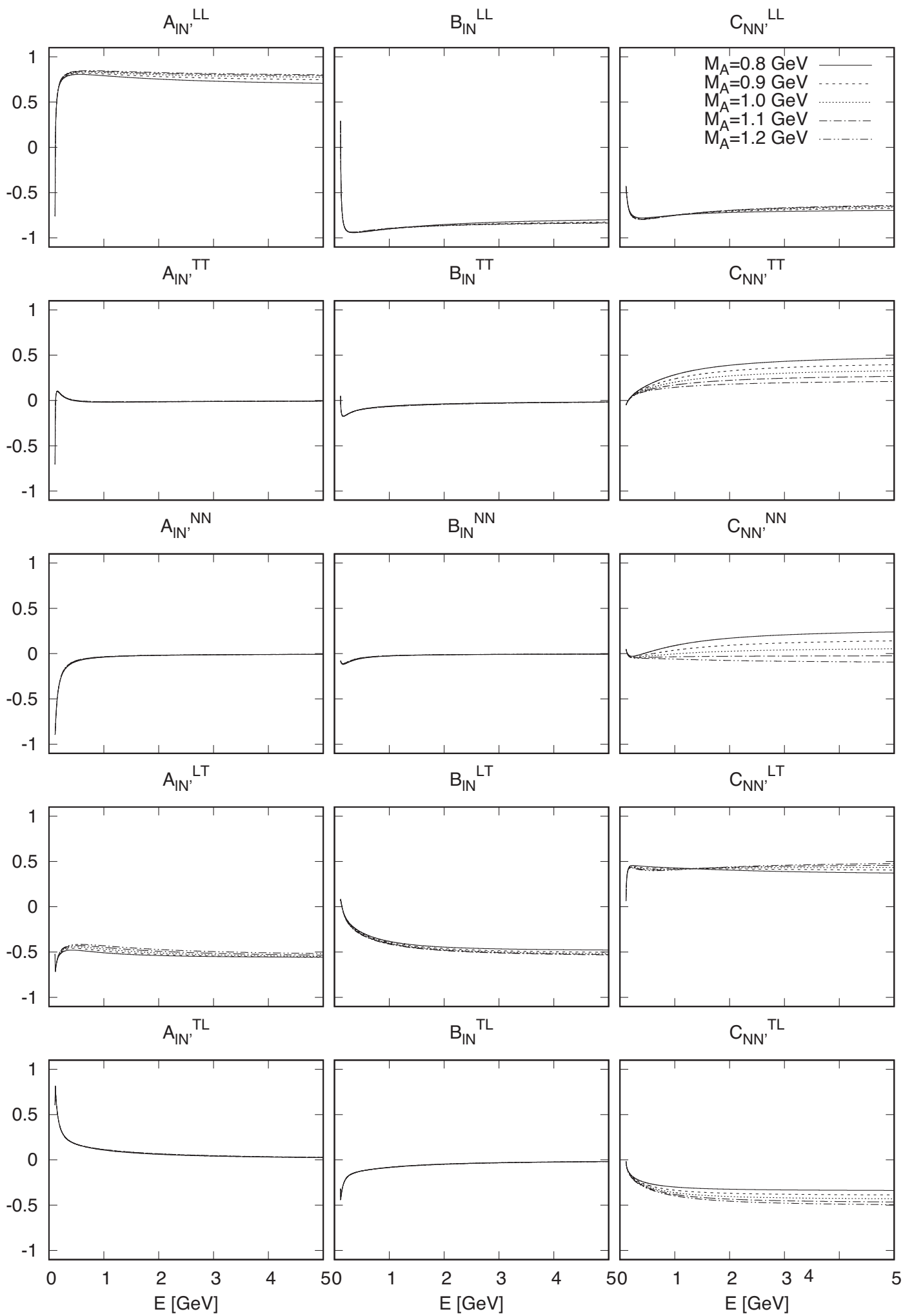

FIG. 5. Energy dependence of the components of double spin asymmetries, $\mathcal{A}_{l N^{\prime}}(E)$ (left column), $\mathcal{B}_{l N^{\prime}}(E)$ (middle column), $\mathcal{C}_{N N^{\prime}}(E)$ (right column), given by the ratios of the total cross sections, see Eqs. (53)-(55), calculated for the CCQE $\nu_{\mu} n$ scattering for $M_{A}=0.8$, $0.9,1.0,1.1,1.2 \mathrm{GeV}$ and $F_{3}^{A}=0$. 

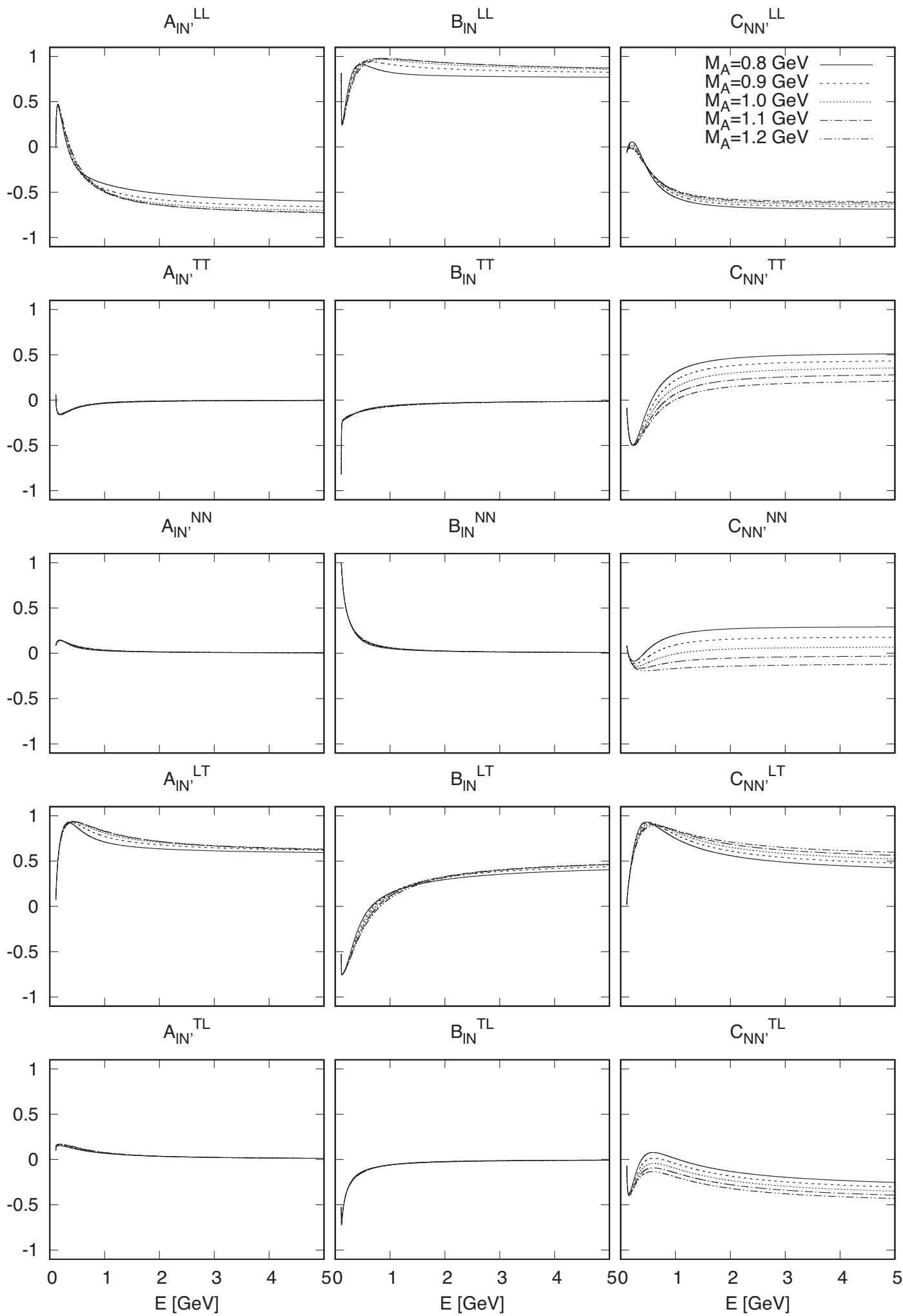

FIG. 6. Caption the same as in Fig. 5 but for the CCQE $\bar{\nu}_{\mu} p$ scattering.

The spin averaged cross section is easily calculated. Indeed, assuming the full form of (15) and reality of the form factors, one gets

$$
\mathcal{I}=\mathcal{I}_{(1)}+\mathcal{I}_{(2)}+\mathcal{I}_{(12)},
$$

where

$\mathcal{I}_{(a)}=\mathrm{L}_{0, \mu \nu} \mathrm{H}_{(a)}^{\mu \nu}\left(s^{N} \rightarrow 0, s^{N^{\prime}} \rightarrow 0\right), \quad a=1,12,2$ 

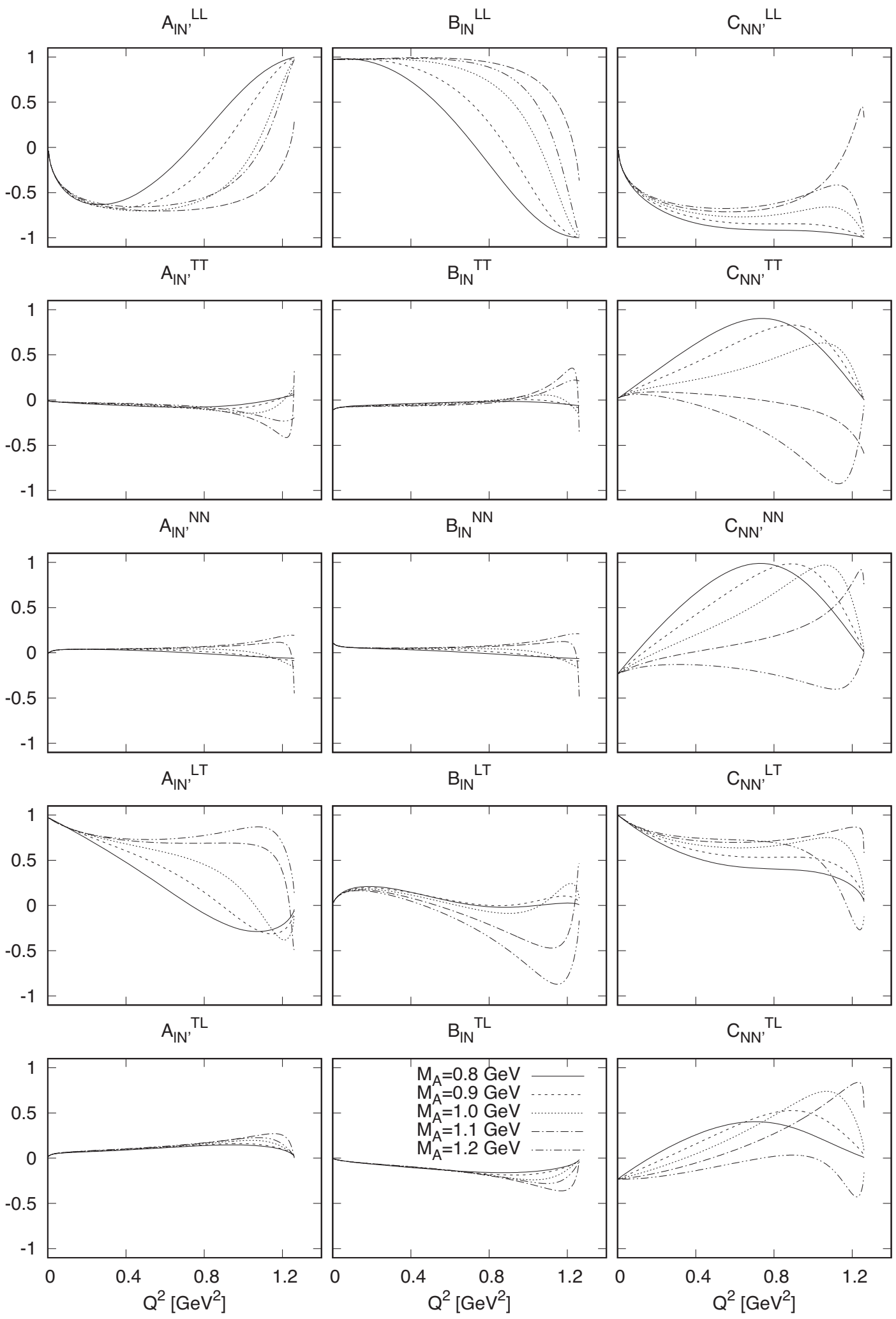

FIG. 7. Dependence of the components of $\mathcal{A}_{l N^{\prime}}\left(E=1 \mathrm{GeV}, Q^{2}\right)$ (left column), $\mathcal{B}_{l N}\left(E=1 \mathrm{GeV}, Q^{2}\right)$ (middle column), and $\mathcal{C}_{N N^{\prime}}(E=$ $1 \mathrm{GeV}, Q^{2}$ ) (right column) on $Q^{2}$; see Eqs. (45)-(47). Results obtained for the CCQE $\bar{\nu}_{\mu} p$ scattering, $M_{A}=0.8,0.9,1.0,1.1,1.2 \mathrm{GeV}$, $F_{3}^{A}=0$, and energy $E=1 \mathrm{GeV}$. 
and

$$
\begin{aligned}
& \mathcal{I}_{(1)}= \frac{1}{4 M^{2}}\left[8 F_{A} F_{P} m^{2} M^{2}\left(t-m^{2}\right)+F_{P}^{2} m^{2} t\left(t-m^{2}\right)+4 F_{A}^{2} M^{2}\left(m^{2}\left(4 M^{2}-m^{2}\right)+s_{u}^{2}-4 M^{2} t+t^{2}\right)\right. \\
&+4 F_{1}^{V^{2}} M^{2}\left(t^{2}-m^{2}\left(m^{2}+4 M^{2}\right)+s_{u}^{2}+4 M^{2} t\right)+8 F_{1}^{V} F_{2}^{V} M^{2}\left(t-m^{2}\right)\left(2 t+m^{2}\right) \\
&\left.+F_{2}^{V 2}\left(t^{3}-4 m^{4} M^{2}-s_{u}^{2} t+\left(4 M^{2}-m^{2}\right) t^{2}\right)-16\left(F_{1}^{V}+F_{2}^{V}\right) F_{A} M^{2} s_{u} t x\right], \\
& \mathcal{I}_{(2)}= \frac{1}{2 M^{2}}\left[m^{2} F_{3}^{V^{2}}\left(5 m^{2} M^{2}-\left(2 m^{2}+5 M^{2}\right) t+2 t^{2}\right)+2 t F_{3}^{A^{2}}\left(4 m^{2} M^{2}-s_{u}{ }^{2}-\left(m^{2}+4 M^{2}\right) t+t^{2}\right)\right], \\
& \mathcal{I}_{(12)}=-4 m^{2} s_{u}\left[\left(F_{1}^{V}+\frac{t}{4 M^{2}} F_{2}^{V}\right) F_{3}^{V}+\left(F_{A}+\frac{t}{4 M^{2}} F_{P}\right) F_{3}^{A}\right] .
\end{aligned}
$$

Above results are consistent with [42].

\section{SPIN OBSERVABLES}

In the most general case, the differential cross section formula has the form

$$
\begin{aligned}
d \sigma= & d \sigma_{0}\left(1+\mathcal{P}_{l}^{\mu} s_{\mu}^{l}+\mathcal{T}_{N}^{\mu} s_{\mu}^{N}+\mathcal{P}_{N^{\prime}}^{\mu} s_{\mu}^{N^{\prime}}+s_{\mu}^{l} s_{\nu}^{N^{\prime}} \mathcal{A}_{l N^{\prime}}^{\mu \nu}\right. \\
& \left.+s_{\mu}^{l} s_{\nu}^{N} \mathcal{B}_{l N}^{\mu \nu}+s_{\mu}^{N} s_{\nu}^{N^{\prime}} \mathcal{C}_{N N^{\prime}}^{\mu \nu}+s_{\mu}^{l} s_{\nu}^{N} s_{\alpha}^{N^{\prime}} \mathcal{D}_{l N N^{\prime}}^{\mu \nu \alpha}\right) .
\end{aligned}
$$

The above expression contains the following seven spin observables [5]:

(i) Recoil polarization asymmetry $\mathcal{P}_{N^{\prime}}^{\mu}$

(ii) Lepton polarization asymmetry $\mathcal{P}_{l}^{\mu}$

(iii) Polarized target asymmetry $\mathcal{T}_{N}^{\mu}$

(iv) Lepton-recoil asymmetry $A_{l N^{\prime}}^{\mu \nu}$

(v) Target-lepton asymmetry $B_{l N}^{\mu \nu}$

(vi) Target-recoil asymmetry $\mathcal{C}_{N N^{\prime}}^{\mu \nu}$

Target-lepton-recoil asymmetry $\mathcal{D}_{l N N^{\prime}}^{\mu \nu \alpha}$

We calculated the spin asymmetry vectors and tensors using the full form of the current (15) and assuming that the

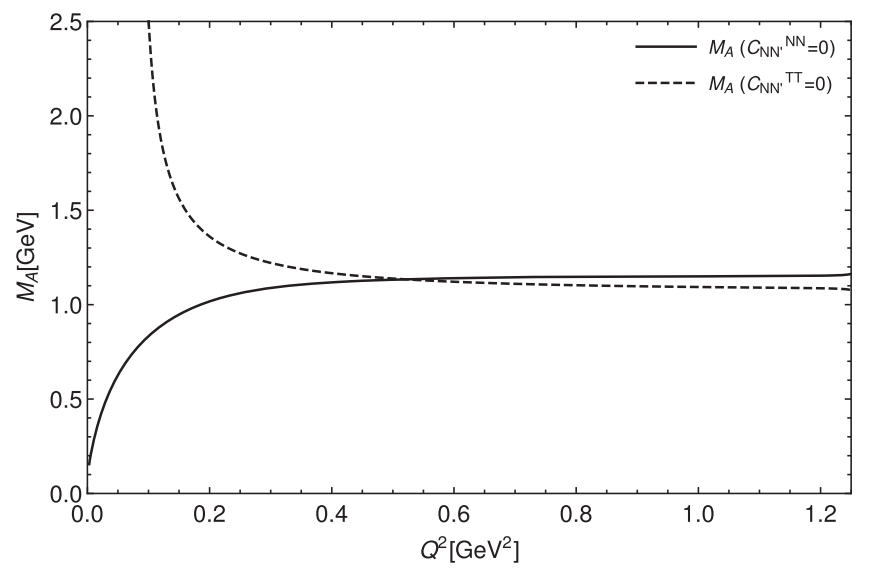

FIG. 8. Axial mass dependence of the roots of the equations: $\mathcal{C}_{N N^{\prime}}^{N N}\left(E=1 \mathrm{GeV}, Q^{2}\right)=0$ (solid line) and $\mathcal{C}_{N N^{\prime}}^{T T}(E=$ $\left.1 \mathrm{GeV}, Q^{2}\right)=0$ (dashed line) obtained for the CCQE $\bar{\nu}_{\mu} p$ scattering. form factors are complex-value functions. The analytic results are given in the Supplemental Material [60].

\section{A. Vector and tensor components of spin asymmetries}

We introduce the spin basis to discuss the physical properties of the spin asymmetries which are as follows:

(i) Basis for the outgoing lepton (see Fig. 1)

$$
\begin{aligned}
& \zeta_{L}^{\mu}=\frac{1}{m}\left(\left|\mathbf{k}^{\prime}\right|, \frac{E_{k^{\prime}} \mathbf{k}^{\prime}}{\left|\mathbf{k}^{\prime}\right|}\right) \quad \zeta_{T}^{\mu}=\left(0, \frac{\mathbf{k}^{\prime} \times(\mathbf{k} \times \mathbf{q})}{\left|\mathbf{k}^{\prime} \times(\mathbf{k} \times \mathbf{q})\right|}\right) \\
& \zeta_{N}^{\mu}=\left(0, \frac{\mathbf{k} \times \mathbf{q}}{|\mathbf{k} \times \mathbf{q}|}\right) \text {, }
\end{aligned}
$$

where $L, T$, and $N$ denote longitudinal to the lepton momentum, transverse to the lepton momentum, and normal to the scattering plane components, respectively.

(ii) Basis for the recoil nucleon

$$
\begin{aligned}
& \xi_{L}^{\mu}=\frac{1}{M}\left(|\mathbf{q}|, \frac{E_{p^{\prime}} \mathbf{q}}{|\mathbf{q}|}\right), \quad \xi_{T}^{\mu}=\left(0, \frac{\mathbf{q} \times(\mathbf{k} \times \mathbf{q})}{|\mathbf{q} \times(\mathbf{k} \times \mathbf{q})|}\right), \\
& \xi_{N}^{\mu}=\left(0, \frac{\mathbf{k} \times \mathbf{q}}{|\mathbf{k} \times \mathbf{q}|}\right),
\end{aligned}
$$

(iii) Basis for the target nucleon

$$
\begin{aligned}
& \chi_{L}^{\mu}=\frac{1}{E}(0, \mathbf{k}), \quad \chi_{T}^{\mu}=\left(0, \frac{\mathbf{k} \times(\mathbf{k} \times \mathbf{q})}{|\mathbf{k} \times(\mathbf{k} \times \mathbf{q})|}\right), \\
& \chi_{N}^{\mu}=\left(0, \frac{\mathbf{k} \times \mathbf{q}}{|\mathbf{k} \times \mathbf{q}|}\right) .
\end{aligned}
$$

We have made the standard choice for the basis vectors for the lepton (31) and recoil nucleon (32). For the target nucleon, the longitudinal vector is parallel to the neutrino momentum, and $\chi_{N}^{\mu}$ is normal to the scattering plane. With this choice of the basis vectors, the axial symmetry around the neutrino momentum $\mathbf{k}$ is maintained; hence, the azimuthal dependence can be easily integrated out. 


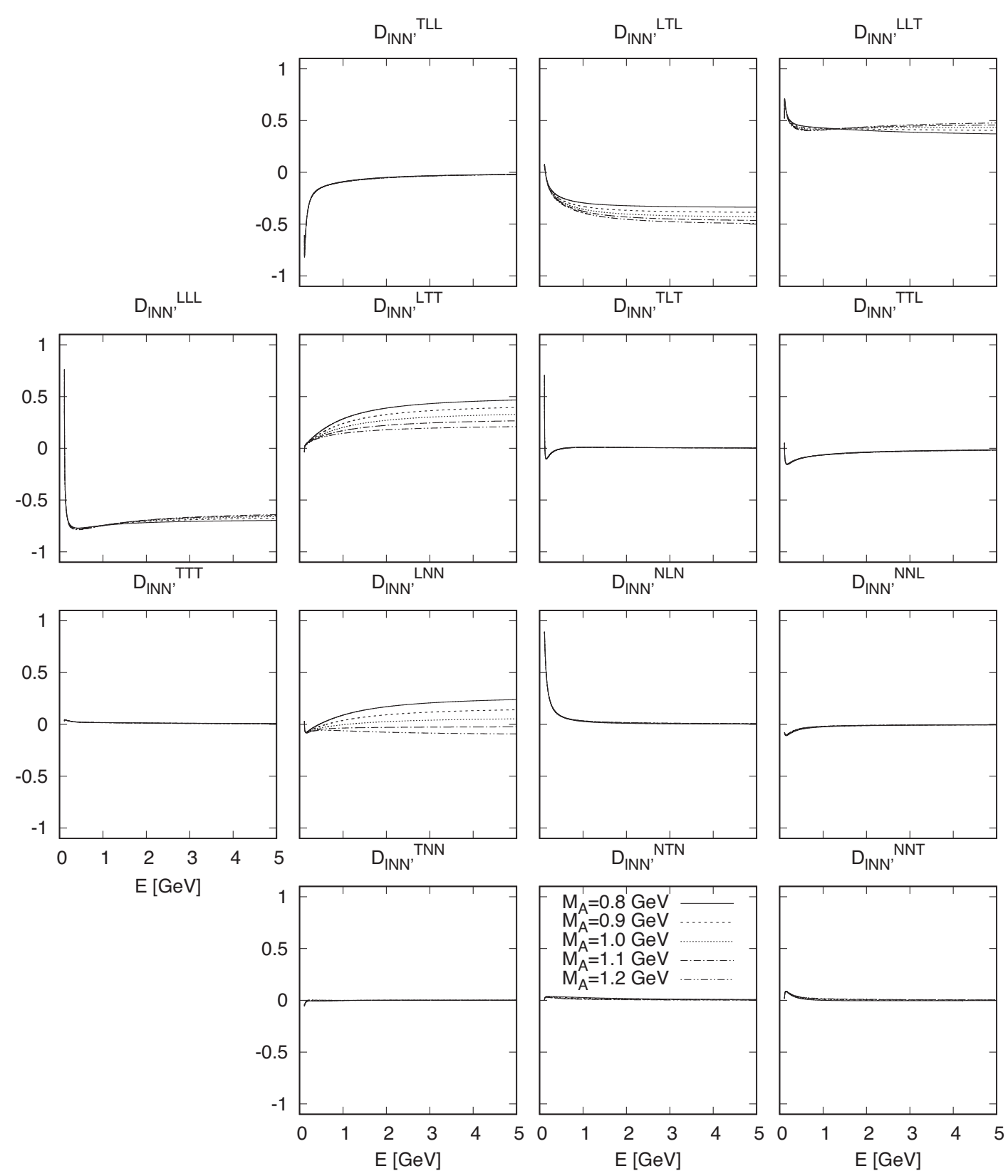

FIG. 9. Energy dependence of the target-recoil-lepton spin asymmetry $\mathcal{D}_{I N N^{\prime}}(E)$, given by the ratios of the total cross sections, see Eqs. (56), calculated for the CCQE $\nu_{\mu} n$ scattering, for $M_{A}=0.8,0.9,1.0,1.1,1.2 \mathrm{GeV}, F_{3}^{A}=0$.

Having the spin basis we calculate the longitudinal (L), transverse $(\mathrm{T})$, and normal $(\mathrm{N})$ components of the polarization asymmetry for the recoil nucleon and the outgoing lepton

$\mathcal{P}_{N^{\prime}}^{X} \equiv \xi_{\mu}^{X} \mathcal{P}_{N^{\prime}}^{\mu} \quad$ and $\quad \mathcal{P}_{l}^{X} \equiv \zeta_{\mu}^{X} \mathcal{P}_{l}^{\mu}, \quad X=L, T, N$,

respectively. If all the form factors are real, then

$$
\mathcal{P}_{N^{\prime}}^{N}=\mathcal{P}_{l}^{N}=0 .
$$

Three independent components of the polarized target asymmetry are given by

$$
\mathcal{T}_{N}^{X} \equiv \chi_{\mu}^{X} \mathcal{T}_{N}^{\mu}, \quad X=L, T, N
$$

For real form factors, $\mathcal{T}_{N}^{N}=0$.

The components of the double spin asymmetry tensor are defined by

$$
\begin{aligned}
\mathcal{B}_{l N}^{X Y} & \equiv \zeta_{\mu}^{X} \chi_{\nu}^{Y} \mathcal{B}_{l N}^{\mu \nu}, \quad \mathcal{A}_{l N^{\prime}}^{X Y}=\zeta_{\mu}^{X} \xi_{\nu}^{Y} \mathcal{A}_{l N^{\prime}}^{\mu \nu}, \\
\mathcal{C}_{N N^{\prime}}^{X Y} & =\chi_{\mu}^{X} \xi_{\nu}^{Y} \mathcal{C}_{N N^{\prime}}^{\mu \nu} .
\end{aligned}
$$

For real form factors, 


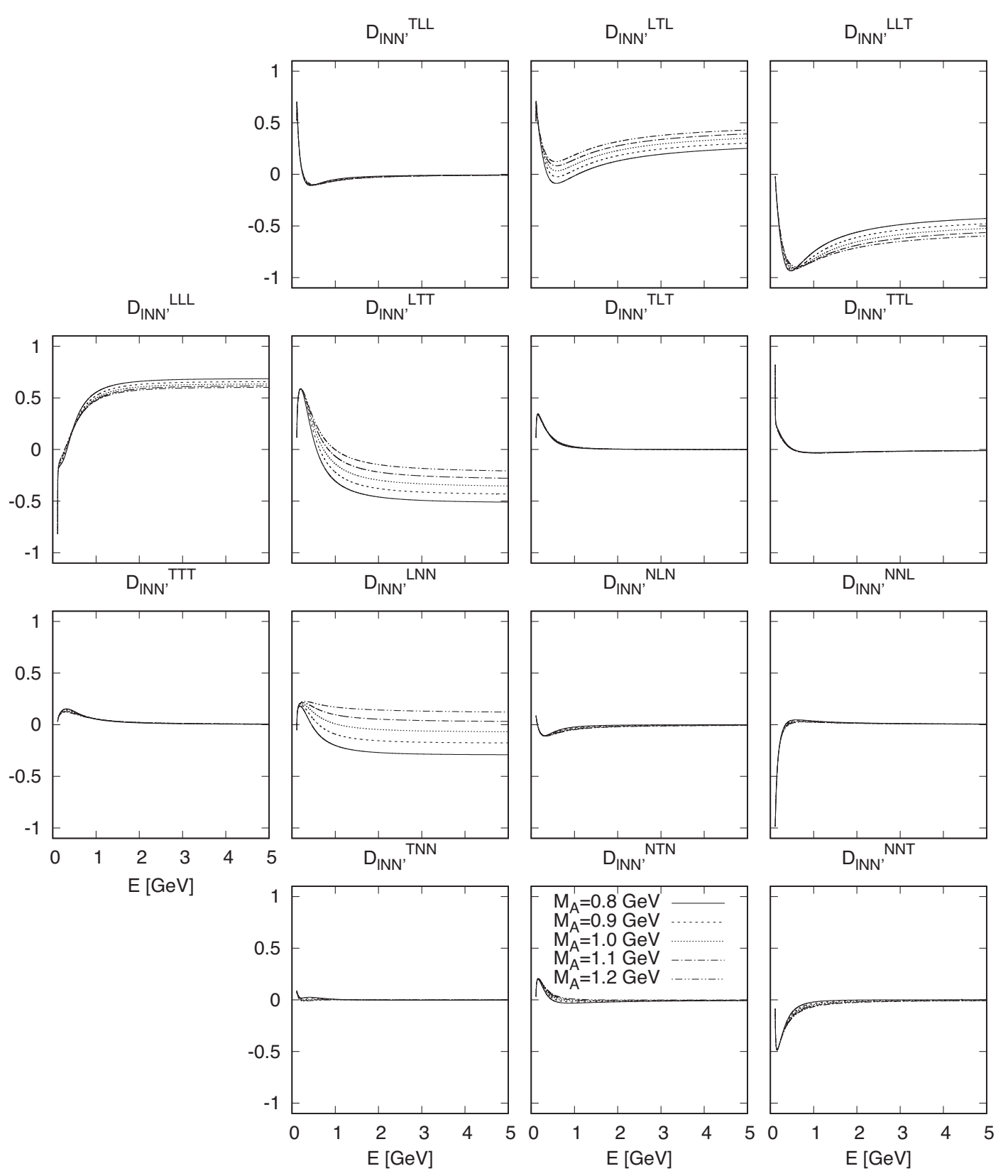

FIG. 10. Caption the same as in Fig. 9 but for the CCQE $\bar{\nu}_{\mu} p$ scattering.

$0=\mathcal{A}_{l N^{\prime}}^{y N}=\mathcal{B}_{l N}^{y N}=\mathcal{C}_{N N^{\prime}}^{y N}=\mathcal{A}_{l N^{\prime}}^{N y}=\mathcal{B}_{l N}^{N y}=\mathcal{C}_{N N^{\prime}}^{N y}$,

$y=L \quad$ or $\quad T$.

Then each tensor has five nonvanishing components.

Eventually, the components of the triple spin asymmetry are defined by

$$
\mathcal{D}_{l N N^{\prime}}^{X Y Z} \equiv \zeta_{\mu}^{X} \chi_{\nu}^{Y} \xi_{\alpha}^{Z} \mathcal{D}_{l N N^{\prime}}^{\mu \nu \alpha}
$$

When the form factors are real, the components given by the contraction of $\mathcal{D}_{l N N^{\prime}}^{\mu \nu \alpha}$ tensor with odd number of normal basis vector vanish, e.g., $\mathcal{D}_{l N N^{\prime}}^{N L T}=0$ or $\mathcal{D}_{l N N^{\prime}}^{N N N}=0$; hence, there are 14 independent components.
Tensor components of the spin asymmetries, calculated assuming that form factors are real and $F_{3}^{V}=0$, are given in the Supplemental Material [60].

Operationally, each asymmetry can be represented by the cross section ratio. For the single spin asymmetries, we introduce the ratio

$$
\begin{aligned}
R\left(d \sigma / d t, S_{X}\right)(E, t) & =\frac{\frac{d \sigma}{d t}\left(S_{X}\right)-\frac{d \sigma}{d t}\left(-S_{X}\right)}{\frac{d \sigma}{d t}\left(S_{X}\right)+\frac{d \sigma}{d t}\left(-S_{X}\right)} \\
& =\frac{\sum_{c= \pm 1} c \frac{d \sigma}{d t}\left(c S_{X}\right)}{\sum_{c= \pm 1} \frac{d \sigma}{d t}\left(c S_{X}\right)}
\end{aligned}
$$




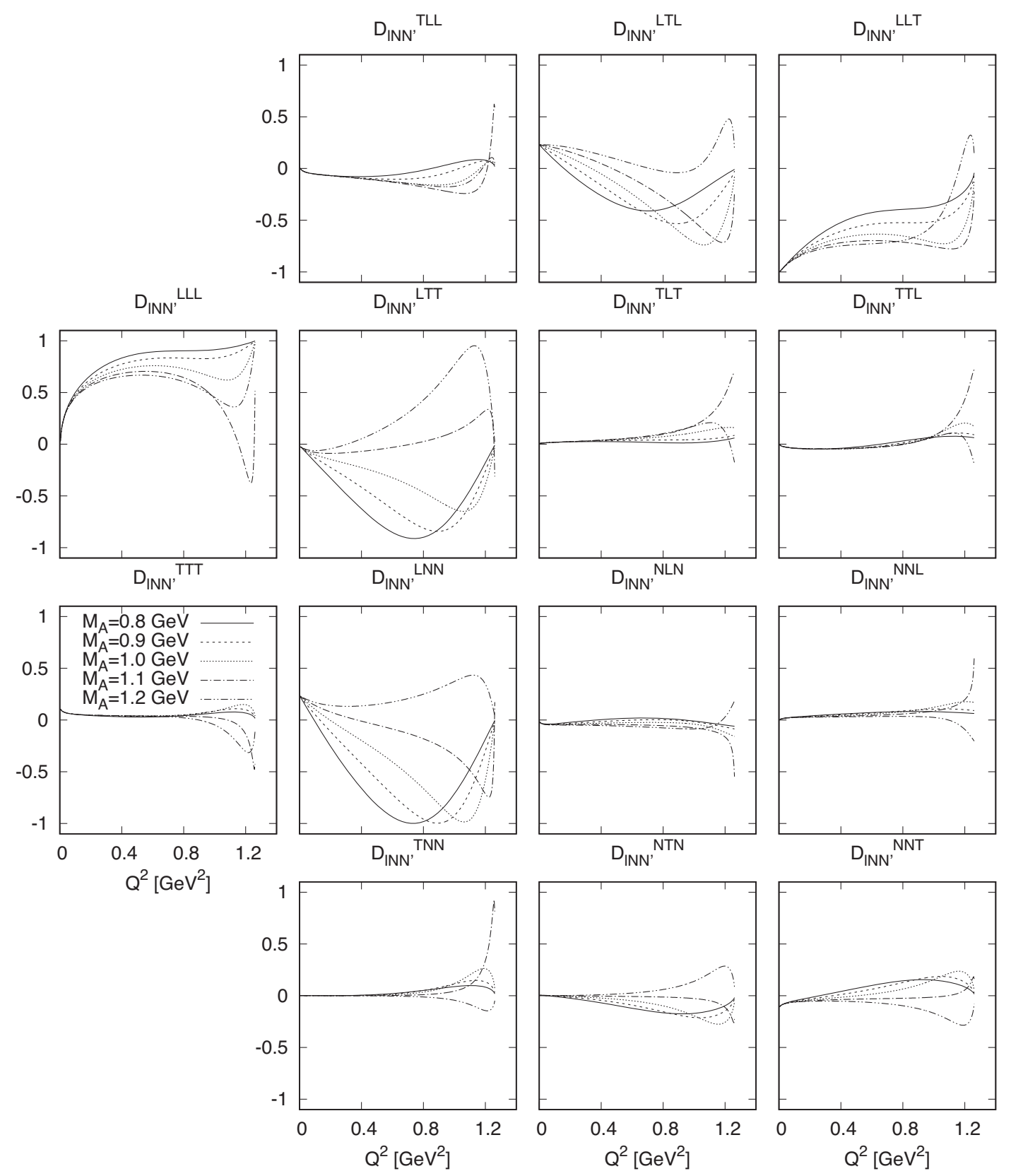

FIG. 11. The dependence of the target-recoil-lepton asymmetry $\mathcal{D}_{I N N^{\prime}}\left(E=1 \mathrm{GeV}, Q^{2}\right)$ on $Q^{2}$, Eq. (48), obtained for the CCQE $\bar{\nu}_{\mu} p$ scattering for $M_{A}=0.8,0.9,1.0,1.1,1.2 \mathrm{GeV}$ and $F_{3}^{A}=0$.

where $S_{X}=\xi_{X}, \zeta_{X}$, or $\chi_{X}$ and $X=L, T, N$. The above ratio schematically can be written as

$$
R\left(d \sigma, S_{X}\right)=\frac{d \sigma(\uparrow)-d \sigma(\downarrow)}{d \sigma(\uparrow)+d \sigma(\downarrow)},
$$

where the arrows indicate the direction of the spin $S_{X}$.

It is easy to see that

$$
\begin{gathered}
\mathcal{P}_{N^{\prime}}^{X}(E, t)=R\left(d \sigma / d t, \xi^{X}\right)(E, t), \\
\mathcal{P}_{l}^{X}(E, t)=R\left(d \sigma / d t, \zeta^{X}\right)(E, t), \\
\mathcal{T}_{N}^{X}(E, t)=R\left(d \sigma / d t, \chi^{X}\right)(E, t) .
\end{gathered}
$$

For the double spin asymmetry, we introduce the ratio $R\left(d \sigma / d t, S_{X}, S_{Y}^{\prime}\right)(E, t) \equiv \frac{\sum_{c_{1}, c_{2}= \pm 1} c_{1} c_{1} \frac{d \sigma}{d t}\left(c_{1} S_{X}, c_{2} S_{Y}^{\prime}\right)}{\sum_{c_{1}, c_{2}= \pm 1} \frac{d \sigma}{d t}\left(c_{1} S_{X}, c_{2} S_{Y}^{\prime}\right)}$, $\rightarrow \frac{\frac{d \sigma}{d t}(\uparrow, \uparrow)+\frac{d \sigma}{d t}(\downarrow, \downarrow)-\frac{d \sigma}{d t}(\uparrow, \downarrow)-\frac{d \sigma}{d t}(\downarrow, \uparrow)}{\frac{d \sigma}{d t}(\uparrow, \uparrow)+\frac{d \sigma}{d t}(\downarrow, \downarrow)+\frac{d \sigma}{d t}(\uparrow, \downarrow)+\frac{d \sigma}{d t}(\downarrow, \uparrow)}$.

Then, 

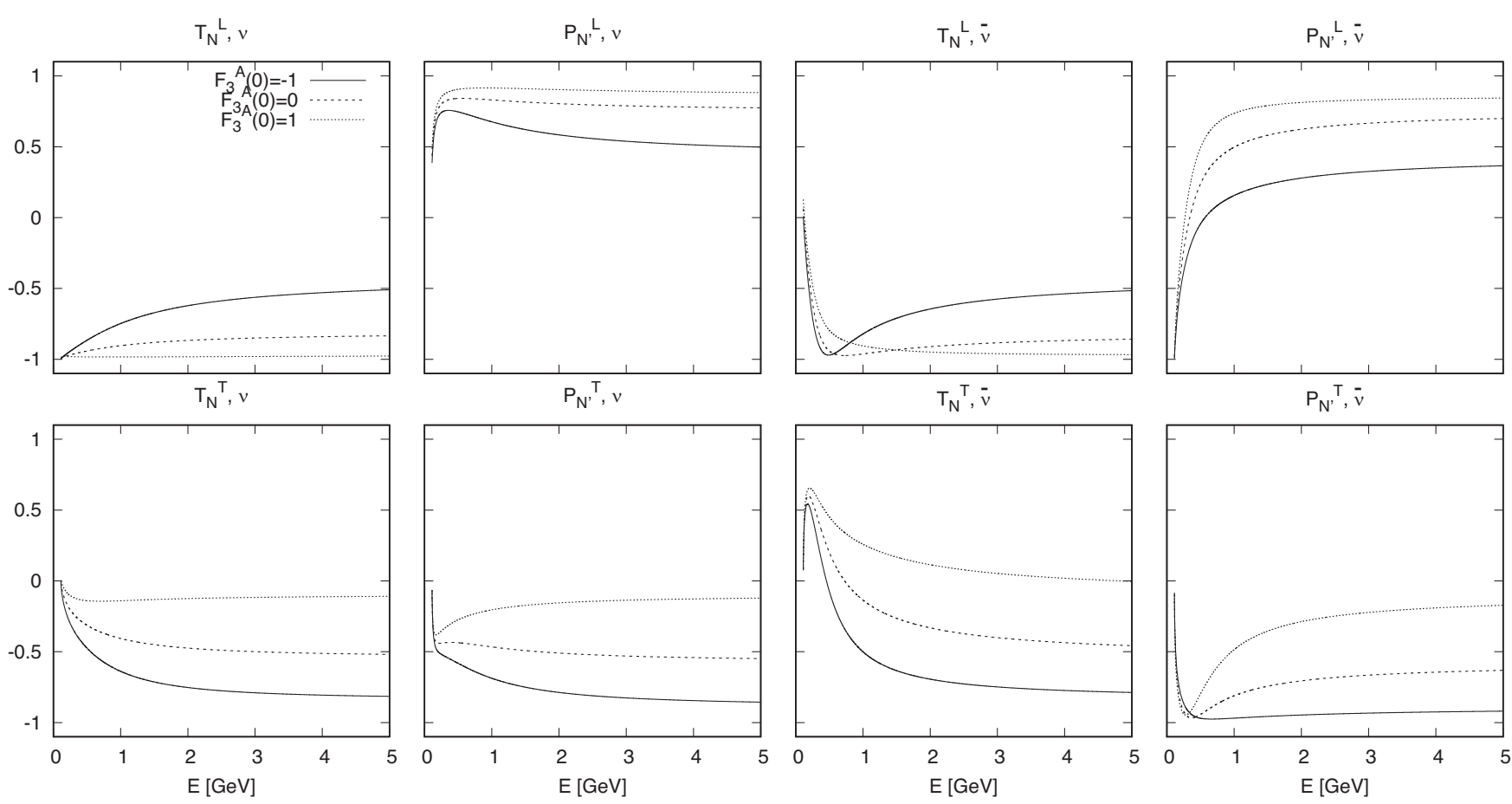

FIG. 12. Dependence of the longitudinal (top row) and transverse (bottom row) components of the polarized target and recoil nucleon asymmetry on $F_{3}^{A}(0)$. The asymmetries are given by the ratios of the total cross sections; see Eqs. (50)-(52). The results obtained for the $\mathrm{CCQE} \nu_{\mu} n$ (the first and the second columns) and $\bar{\nu}_{\mu} p$ (the third and fourth columns) interactions, $M_{A}=1.0 \mathrm{GeV}$ and $F_{3}^{A}(0)=-1,0,1$.

$$
\begin{gathered}
\mathcal{A}_{l N^{\prime}}^{X Y}(E, t)=R\left(d \sigma / d t, \zeta^{X}, \xi^{Y}\right)(E, t), \\
\mathcal{B}_{l N}^{X Y}(E, t)=R\left(d \sigma / d t, \zeta^{X}, \chi^{Y}\right)(E, t), \\
\mathcal{C}_{N N^{\prime}}^{X Y}(E, t)=R\left(d \sigma / d t, \chi^{X}, \xi^{Y}\right)(E, t) .
\end{gathered}
$$

For the triple spin asymmetry, we introduce the ratio

$$
\begin{aligned}
\mathcal{D}_{l N N^{\prime}}^{X Y Z}(E, t) & \equiv R\left(d \sigma / d t, \zeta^{X}, \xi^{Y}, \chi^{Z}\right)(E, t) \\
& =\frac{\sum_{c_{1}, c_{2}, c_{3}= \pm 1} c_{1} c_{2} c_{3} \frac{d \sigma}{d t}\left(c_{1} \zeta_{X}, c_{2} \chi_{Y}, c_{3} \xi_{Z}\right)}{\sum_{c_{1}, c_{2}, c_{3}= \pm 1} \frac{d \sigma}{d t}\left(c_{1} \zeta_{X}, c_{2} \chi_{Y}, c_{3} \xi_{Z}\right)} .
\end{aligned}
$$

We present also the asymmetries given by the ratios of the total cross sections; in this case,

$R\left(\sigma, S_{X}\right)(E) \equiv \frac{\sum_{c= \pm 1} c \sigma\left(c S_{X}\right)}{\sum_{c= \pm 1} \sigma\left(c S_{X}\right)}, \quad \sigma=\int d t \frac{d \sigma}{d t}$.

Similar ratios are defined for the double and the triple spin asymmetries.

In order to unify the notation, the ratios of the total cross sections are denoted similarly as the corresponding ratios of $d \sigma / d t$ cross sections, namely

$$
\mathcal{P}_{N^{\prime}}^{X}(E) \rightarrow R\left(\sigma, \xi^{X}\right)(E)
$$

$$
\begin{gathered}
\mathcal{P}_{l}^{X}(E) \rightarrow R\left(\sigma, \zeta^{X}\right)(E), \\
\mathcal{T}_{N}^{X}(E) \rightarrow R\left(\sigma, \chi^{X}\right)(E), \\
\mathcal{A}_{l N^{\prime}}^{X Y}(E) \rightarrow R\left(\sigma, \zeta^{X}, \xi^{Y}\right)(E), \\
\mathcal{B}_{l N}^{X Y}(E) \rightarrow R\left(\sigma, \zeta^{X}, \chi^{Y}\right)(E), \\
\mathcal{C}_{N N^{\prime}}^{X Y}(E) \rightarrow R\left(\sigma, \chi^{X}, \xi^{Y}\right)(E), \\
\mathcal{D}_{l N N^{\prime}}^{X Y Z}(E) \rightarrow R\left(\sigma, \zeta^{X}, \xi^{Y}, \chi^{Z}\right)(E) .
\end{gathered}
$$

\section{NUMERICAL RESULTS AND DISCUSSION}

We start the discussion of the results from the analyses of the sensitivity of spin asymmetries to the axial contribution. The numerical calculations are made assuming that

(i) all form factors are real;

(ii) the SCC vanishes, hence $F_{3}^{V}=0$ and $F_{3}^{A}=0$;

(iii) the axial form factor is given by the dipole parametrization,

$$
F_{A}(t)=\frac{g_{A}}{\left(1-\frac{t}{M_{A}^{2}}\right)^{2}},
$$

where $M_{A}$ is the axial mass, which for the default value takes $1 \mathrm{GeV}$, and $g_{A}=1.2723 \pm 0.0023$ [64]. 

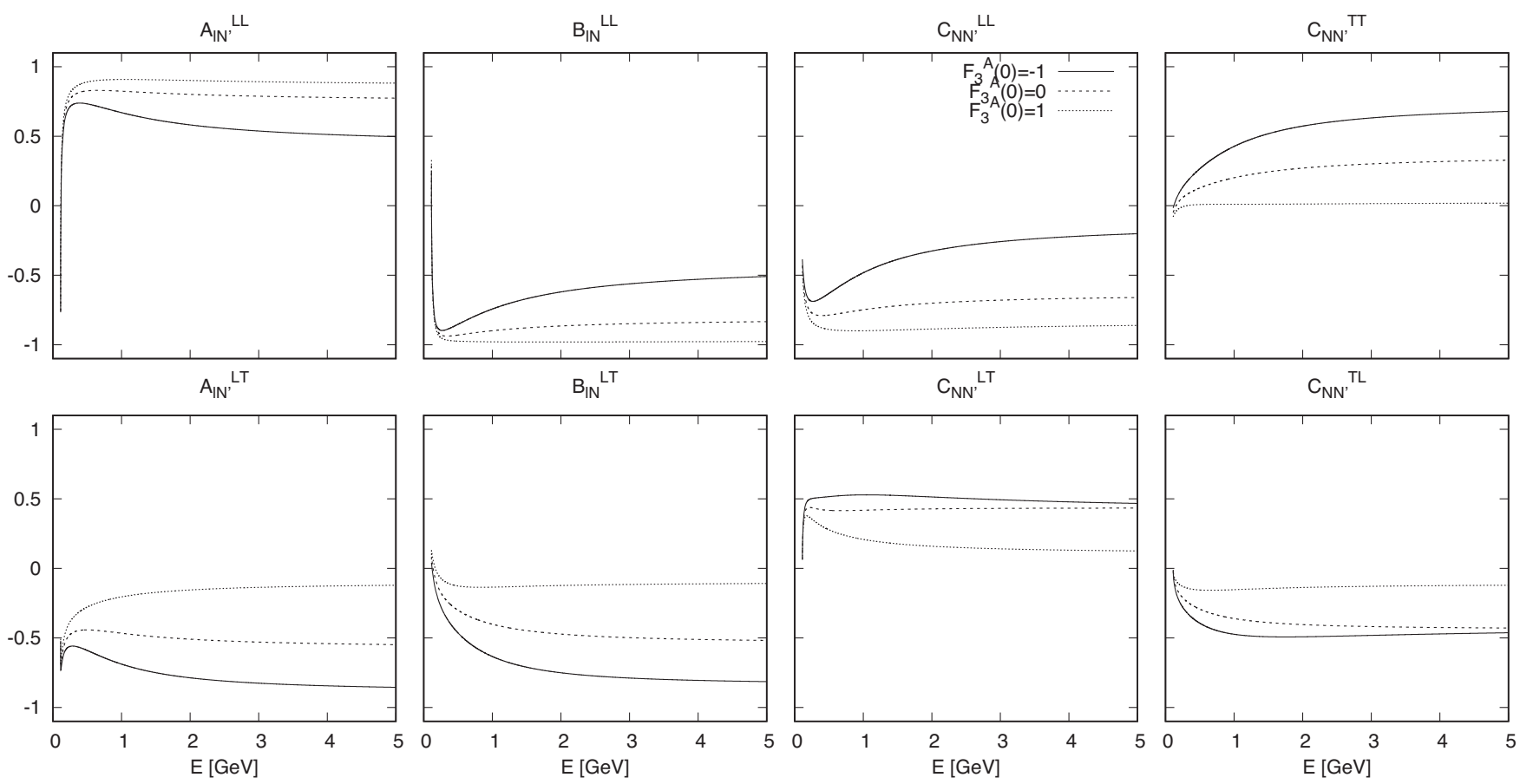

FIG. 13. Energy dependence of the components of double spin asymmetries: $\mathcal{A}_{l N^{\prime}}(E), \mathcal{B}_{l N^{\prime}}(E), \mathcal{C}_{N N^{\prime}}(E)$, given by the ratios of the total cross sections, see Eqs. (53)-(55), calculated for the CCQE $\nu_{\mu} n$ scattering with $M_{A}=1.0 \mathrm{GeV}$ and for $F_{3}^{A}(0)=-1,0$, or 1 .

We present the numerical results for two types of asymmetries, namely, given by the ratios of total and $d \sigma / d t$ cross sections. The first depend on the energy $E$, whereas the latter is a function of $E$ and $Q^{2}=-t$.

In the preanalyses, we considered also the parametrization of $F_{A}$ defined by the sum of two monopoles [27]. But it turned out that effectively this parametrization works very similarly as the dipole with $M_{A}=1.4 \mathrm{GeV}$. Therefore, in this work, we present the results only for $F_{A}$ given by Eq. (57). To mimic different shapes of $F_{A}$, the value of $M_{A}$ is varied, namely, $M_{A}=0.8,0.9,1.0,1.1$, and $1.2 \mathrm{GeV}$.

In Figs. 2 and 3, we present the dependence of the components of the single spin asymmetries on the energy. Here, the asymmetries are given by the ratios of the total cross sections; see Eqs. (50)-(52). We present the plots for both the CCQE processes, namely, $\nu_{\mu} n$ and $\bar{\nu}_{\mu} p$ interactions. The impact of the axial contribution on the recoil nucleon and the lepton polarizations is discussed in Refs. [32,46]. Our results confirm the conclusions of previous investigations, namely, the outgoing muon polarization, for both types of processes, weakly depends on the shape of the axial form factor. But the recoil polarization, for the $\bar{\nu}_{\mu} p$ scattering, is sensitive to $F_{A}$.

As has been mentioned in the introduction, the polarized target asymmetry has been not studied yet. Similarly, as for $\mathcal{P}_{N^{\prime}}$, the longitudinal and transverse components of the $\mathcal{T}_{N}$, calculated for $\bar{\nu}_{\mu} p$ scattering, are sensitive to the axial contribution. The change of the functional $Q^{2}$ dependence of $F_{A}$ results in distortion of the shape of the components of $\mathcal{T}_{N}$. This effect is the strongest for the antineutrino scattering for large $Q^{2}$ values; see Fig. 4.

The double spin asymmetries given by the ratios of total cross sections are plotted in Figs. 5 and 6. Among three observables $\mathcal{A}_{l N^{\prime}}(E), \mathcal{B}_{l N}(E)$, and $\mathcal{C}_{N N^{\prime}}(E)$, the last onethe target-recoil double spin asymmetry is the most sensitive to the axial contribution. In particular, this effect is visible for the components $\mathcal{C}_{N N^{\prime}}^{T T}$ and $\mathcal{C}_{N N^{\prime}}^{N N}$. Indeed, a reduction of the axial mass leads to an increasing of the amplitude of the components $\mathcal{C}_{N N^{\prime}}^{T T}$ and $\mathcal{C}_{N N^{\prime}}^{N N}$ and a change of their signs from positive to negative. Indeed, for instance, for $\bar{\nu}_{\mu} p$ scattering at fixed energy $(E=1 \mathrm{GeV})$ for large values of $M_{A}$, the observables $\mathcal{C}_{N N^{\prime}}^{T T}\left(E, Q^{2}\right)$ and $\mathcal{C}_{N N^{\prime}}^{N N}\left(E, Q^{2}\right)$ are negative, whereas for large $M_{A}$, they take the positive values in full $Q^{2}$ range; see Fig. 7. Hence, the asymmetries $\mathcal{C}_{N N^{\prime}}^{T T}$ and $\mathcal{C}_{N N^{\prime}}^{N N}$ are well-suited observables for estimation of the value of the $M_{A}$ and for studies of the $Q^{2}$ dependence of $F_{A}$. For an illustration of this property, let us present the numerical solutions of two equations, obtained for the CCQE $\bar{\nu}_{\mu} p$ scattering,

$$
\begin{aligned}
& 0=\mathcal{C}_{N N^{\prime}}^{T T}\left(E=1 \mathrm{GeV}, Q^{2}\right), \\
& 0=\mathcal{C}_{N N^{\prime}}^{N N}\left(E=1 \mathrm{GeV}, Q^{2}\right) .
\end{aligned}
$$

The roots of the above equations depend on the value of $M_{A}$; see Fig. 8. Notice that for too small values of the axial mass the first equation has no solution, whereas for too large values of $M_{A}$, the second equation has no roots. 

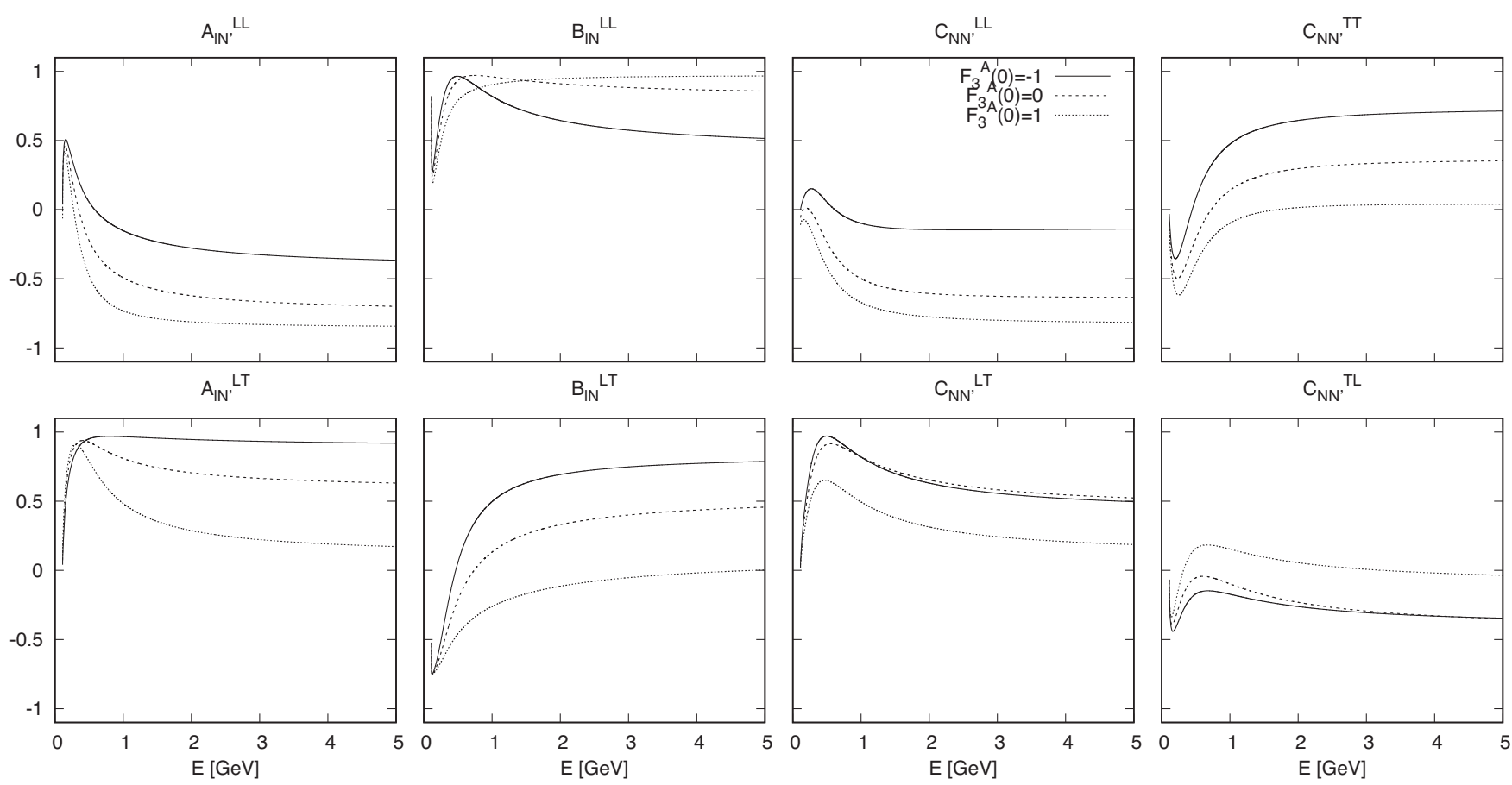

FIG. 14. Caption the same as in Fig. 13 but for the CCQE $\bar{\nu}_{\mu} p$ scattering.

Moreover, for the value of $M_{A} \approx 1.0 \mathrm{GeV}$, which is suggested by the recent neutrino scattering data analyses, the roots of Eq. (59) monotonically depend on $M_{A}$.

The triple spin asymmetries, $\mathcal{D}_{I N N^{\prime}}(E)$, are shown in Figs. 9 and 10, respectively. Similarly, as for the tensor
$\mathcal{C}_{N N^{\prime}}$, the spin asymmetry components calculated for $\bar{\nu}_{\mu} p$ scattering are more sensitive to the axial form factor shape than the components calculated for neutrino scattering. In Fig. 11, we plot the $Q^{2}$ dependence of the components of the $\mathcal{D}_{l N N^{\prime}}\left(E=1 \mathrm{GeV}, Q^{2}\right)$. The most sensitive to the shape
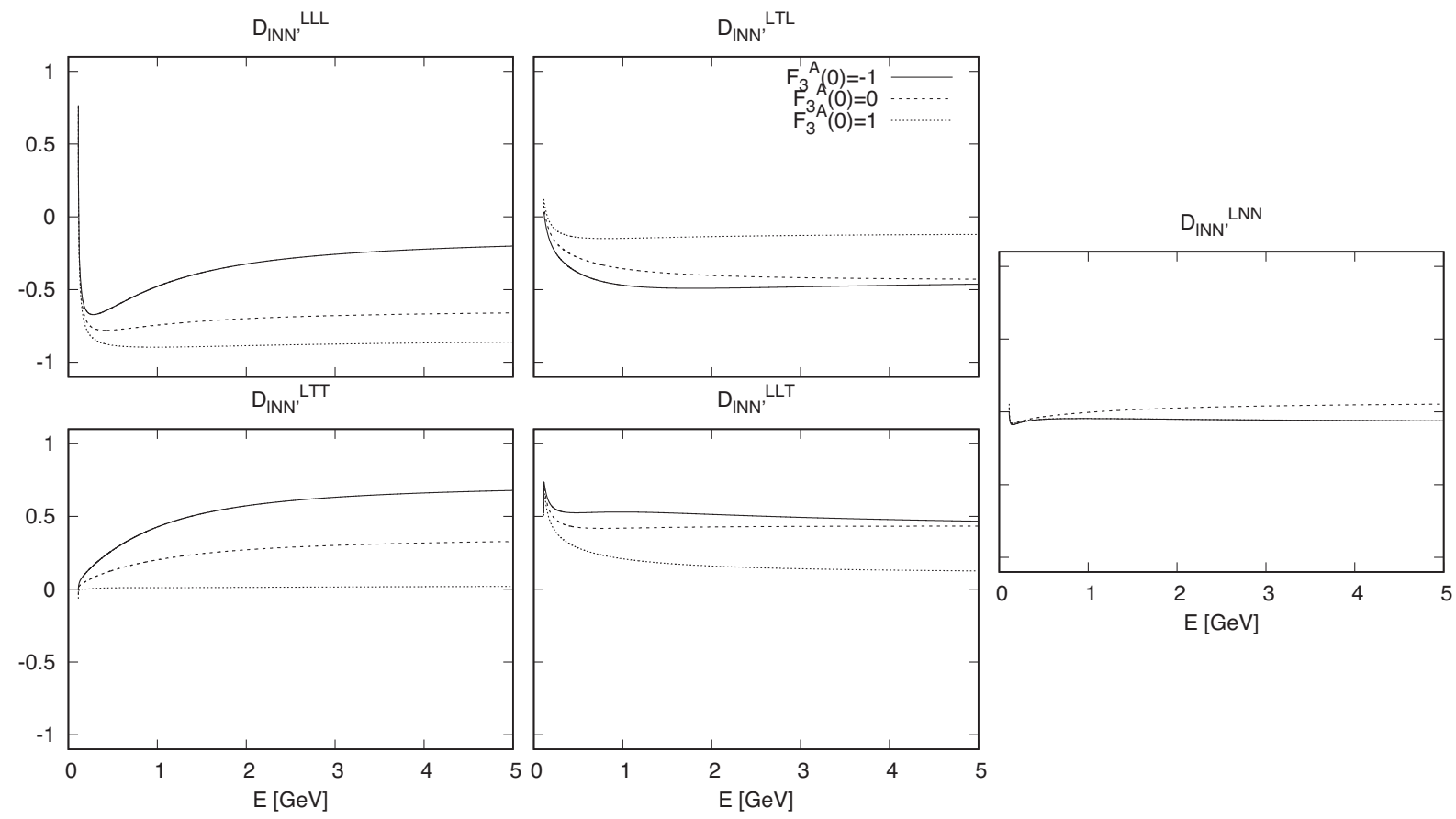

FIG. 15. Energy dependence of the components of triple spin asymmetry $\mathcal{D}_{I N N^{\prime}}(E)$, given by the ratios of the total cross sections, see Eq. (56), calculated for the CCQE $\nu_{\mu} n$ scattering with $M_{A}=1.0 \mathrm{GeV}$ and for $F_{3}^{A}(0)=-1,0$, or 1 . 


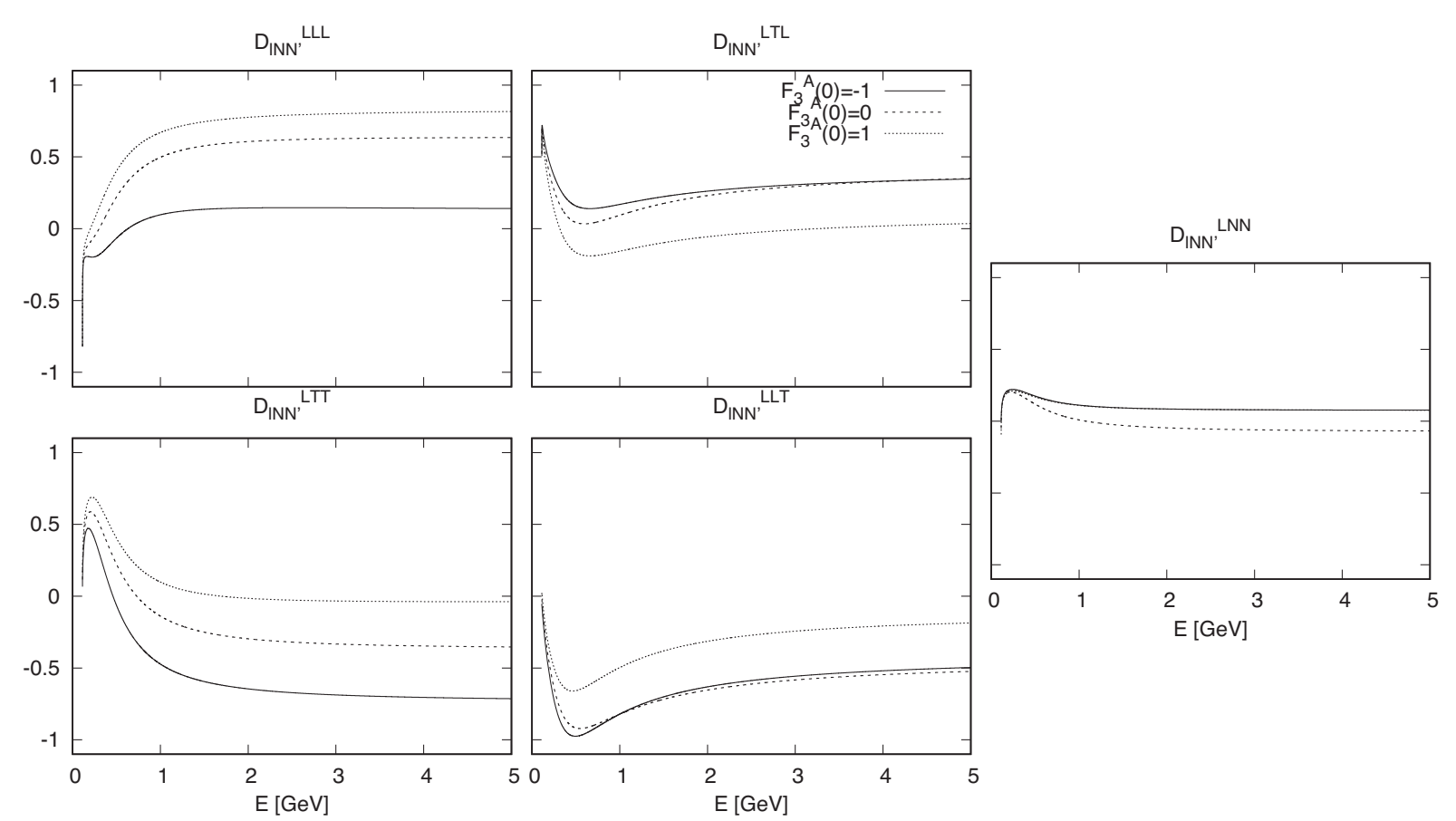

FIG. 16. Caption the same as in Fig. 15 but for the CCQE $\bar{\nu}_{\mu} p$ scattering.

of the axial form factor are the asymmetries obtained from the contraction of the longitudinal component of the lepton spin vector with the tensor $\mathcal{D}_{l N N^{\prime}}^{\mu \nu \alpha}$, namely, the components $\mathcal{D}_{l N N^{\prime}}^{L N N}, \mathcal{D}_{l N N^{\prime}}^{L T T}$, and $\mathcal{D}_{l N N^{\prime}}^{L T L}$. Analogically, as for the double spin asymmetry case, the sign of these components depends on the value of $M_{A}$.

The SCC contribution in the lepton and the recoil spin asymmetries was studied in Ref. [32]. In our analysis, we additionally consider polarized target asymmetry, double spin asymmetries, and triple spin asymmetry. In this part of the work, the numerical results are obtained assuming that

(i) all form factors are real;

(ii) $F_{3}^{A}$ form factor is given by [32]

$$
F_{A}^{3}(t)=\frac{F_{A}^{3}(0)}{\left(1-t / M_{A}^{2}\right)^{2}},
$$

where $F_{A}^{(0)}$ is an unknown parameter and for numerical calculations $F_{A}^{3}(0)=-1,0$, and 1 ;

(iii) $F_{3}^{V}=0$;

(iv) $M_{A}=1.0 \mathrm{GeV}$ in (57) and (60).

To reduce the number of figures, we show only the components of the spin asymmetries which are sensitive to change of $F_{A}^{3}$. In Fig. 12, the single spin asymmetries are plotted, whereas in Figs. 13 and 14, we show the double spin asymmetries, as a function of energy, calculated for $\nu_{\mu} n$ and $\bar{\nu}_{\mu} p$ interactions, respectively. The triple spin asymmetries are plotted in Figs. 15 and 16. Concluding this part, we state that outgoing muon spin asymmetry is insensitive to the SCC contribution, whereas other single, double, and triple spin asymmetries are good observables for studying the signal from the nonstandard interactions.

\section{SUMMARY}

The spin observables in the CCQE $\nu_{\mu} n$ and $\bar{\nu}_{\mu} p$ interactions have been studied. We considered seven spin asymmetries. Five of them, namely, the polarized target asymmetry, double and triple asymmetries have been not discussed yet. All asymmetries were calculated analytically; they are given, in the covariant form, in Sec. I of the Supplemental Material [60]. To discuss the physical properties of the asymmetries, we introduced the spin basis vectors and the physical components of the asymmetries were computed and given in Sec. II of the Supplemental Material [60]. Eventually, we studied the dependence of the spin asymmetries on the axial and SCC contributions.

We showed that the target-recoil asymmetry, $\mathcal{C}_{N N^{\prime}}$, as well as the lepton-target-recoil asymmetry, $\mathcal{D}_{l N N^{\prime}}$, are wellsuited observables for studying the axial nucleon form factor. Indeed, the sign and magnitude of the components $\mathcal{C}_{\mathcal{N} \mathcal{N}^{\prime}}^{\mathcal{N}}, \mathcal{C}_{\mathcal{N N}^{\prime}}^{\mathcal{T}}$, depend strongly on the axial mass value. Similar effect is found for the components $\mathcal{D}_{l N N^{\prime}}^{L N N}, \mathcal{D}_{l N N^{\prime}}^{L T T}$, and $\mathcal{D}_{l N N^{\prime}}^{L T L}$. Eventually, we showed that all the spin asymmetries, except the lepton polarization asymmetry, are promising observables for investigation of the nonstandard interactions in the neutrino scattering processes.

\section{ACKNOWLEDGMENTS}

The calculations have been carried out in Wroclaw Centre for Networking and Supercomputing (http://www .wcss.wroc.pl), Grant No. 268. A part of the algebraic calculations presented has been performed using FORM language [65] and FeynCalc package [66,67]. 


\section{APPENDIX: CONVENTION, NORMALIZATION, AND KINEMATICS}

We work with the metric tensor

$$
g_{\mu \nu}=\operatorname{diag}(1,-1,-1,-1) .
$$

For the Levi-Civita tensor, we keep the normalization so that

$$
\epsilon_{0123}=1
$$

The Dirac field of 1/2-particle of mass $M$, momentum $p$, and spin $s$ is normalized so that

$$
\begin{aligned}
\bar{u}(p, s) u\left(p, s^{\prime}\right) & =2 M \delta_{s s^{\prime}}, \\
u(p, s) \bar{u}(p, s) & =\frac{1}{2}\left(1+\gamma_{5} \not\right)(\not p+M) .
\end{aligned}
$$

[1] C. Bourrely, J. Soffer, and E. Leader, Phys. Rep. 59, 95 (1980).

[2] E. Leader, Spin in Particle Physics, Cambridge Monographs on Particle Physics, Nuclear Physics and Cosmology (Cambridge University Press, Cambridge, United Kingdom, 2001).

[3] H. Fraas, Z. Phys. 234, 472 (1970).

[4] M. J. Alguard et al., Phys. Rev. Lett. 37, 1258 (1976).

[5] C. G. Fasano, F. Tabakin, and B. Saghai, Phys. Rev. C 46, 2430 (1992).

[6] K. Nakayama, J. Phys. G 46, 105108 (2019).

[7] A. I. Akhiezer and M. Rekalo, Dokl. Akad. Nauk Ser. Fiz. 180, 1081 (1968) [Sov. Phys. Dokl. 13, 572 (1968)], https:// inspirehep.net/literature/53475.

[8] A. I. Akhiezer and M. Rekalo, Fiz. Elem. Chastits At. Yadra 4, 662 (1973) [Sov. J. Part. Nucl. 4, 277 (1974)], https:// inspirehep.net/literature/95715.

[9] R. G. Arnold, C. E. Carlson, and F. Gross, Phys. Rev. C 23, 363 (1981).

[10] T. W. Donnelly and A. S. Raskin, Ann. Phys. (N.Y.) 169, 247 (1986).

[11] A. Afanasev, P. G. Blunden, D. Hasell, and B. A. Raue, Prog. Part. Nucl. Phys. 95, 245 (2017).

[12] H. Arenhövel, Phys. Rev. C 99, 055502 (2019).

[13] U. Mosel, Annu. Rev. Nucl. Part. Sci. 66, 171 (2016).

[14] L. Alvarez-Ruso et al., Prog. Part. Nucl. Phys. 100, 1 (2018).

[15] F. Sánchez, New neutrino cross-section measurements in t2k (2018), https://doi.org/10.5281/zenodo.1295707.

[16] M. Sanchez, Nova results and prospects (2018), https:// doi.org/10.5281/zenodo.1286758.

[17] V. Bernard, L. Elouadrhiri, and U.-G. Meissner, J. Phys. G 28, R1 (2002).

[18] T. Katori and M. Martini, J. Phys. G 45, 013001 (2018).

[19] W. A. Mann et al., Phys. Rev. Lett. 31, 844 (1973).

[20] S. J. Barish et al., Phys. Rev. D 16, 3103 (1977).

[21] K. L. Miller et al., Phys. Rev. D 26, 537 (1982).

[22] N. J. Baker, A. M. Cnops, P. L. Connolly, S. A. Kahn, H. G. Kirk, M. J. Murtagh, R. B. Palmer, N. P. Samios, and M. Tanaka, Phys. Rev. D 23, 2499 (1981).

[23] T. Kitagaki, H. Yuta, S. Tanaka, A. Yamaguchi, K. Abe et al., Phys. Rev. D 34, 2554 (1986).
[24] T. Kitagaki et al., Phys. Rev. D 42, 1331 (1990).

[25] T. Kitagaki et al., Phys. Rev. D 28, 436 (1983).

[26] A. Bodek, S. Avvakumov, R. Bradford, and H. S. Budd, Eur. Phys. J. C 53, 349 (2008).

[27] J. E. Amaro and E. Ruiz Arriola, Phys. Rev. D 93, 053002 (2016).

[28] A. S. Meyer, M. Betancourt, R. Gran, and R. J. Hill, Phys. Rev. D 93, 113015 (2016).

[29] L. Alvarez-Ruso, K. M. Graczyk, and E. Saul-Sala, Phys. Rev. C 99, 025204 (2019).

[30] G. D. Megias, S. Bolognesi, M. B. Barbaro, and E. TomasiGustafsson, Phys. Rev. C 101, 025501 (2020).

[31] S. Weinberg, Phys. Rev. 112, 1375 (1958).

[32] A. Fatima, M. Sajjad Athar, and S. K. Singh, Phys. Rev. D 98, 033005 (2018).

[33] L. A. Ahrens et al., Phys. Lett. B 202, 284 (1988).

[34] S. V. Belikov et al., Z. Phys. A 320, 625 (1985).

[35] D. H. Wilkinson, Eur. Phys. J. A 7, 307 (2000).

[36] K. M. Graczyk and B. E. Kowal, Phys. Rev. D 97, 013001 (2018).

[37] K. M. Graczyk and B. E. Kowal, Acta Phys. Pol. B 48, 2219 (2017).

[38] K. M. Graczyk and B. E. Kowal, Phys. Rev. D 99, 053002 (2019).

[39] S. L. Adler, Nuovo Cimento (1955-1965) 30, 1020 (1963).

[40] A. Pais, Ann. Phys. (N.Y.) 63, 361 (1971).

[41] M. M. Block, in Symmetries in Elementary Particle Physics: International School of Physics Ettore Majorana, Erice, Italy, 1964 (1965), p. 341, https://doi.org/10.1016/B978-14832-5648-1.50017-8.

[42] C. H. Llewellyn Smith, Phys. Rep. 3, 261 (1972).

[43] K. Hagiwara, K. Mawatari, and H. Yokoya, Nucl. Phys. B668, 364 (2003); B701, 405(E) (2004).

[44] K. S. Kuzmin, V. V. Lyubushkin, and V. A. Naumov, Nucl. Phys. B, Proc. Suppl. 139, 154 (2005).

[45] K. S. Kuzmin, V. V. Lyubushkin, and V. A. Naumov, Mod. Phys. Lett. A 19, 2919 (2004).

[46] S. M. Bilenky and E. Christova, Phys. Part. Nucl. Lett. 10, 651 (2013).

[47] S. M. Bilenky and E. Christova, J. Phys. G 40, 075004 (2013).

[48] K. M. Graczyk, Nucl. Phys. A748, 313 (2005). 
[49] M. Valverde, J. E. Amaro, J. Nieves, and C. Maieron, Phys. Lett. B 642, 218 (2006).

[50] J. E. Sobczyk, N. Rocco, and J. Nieves, Phys. Rev. C 100, 035501 (2019).

[51] N. Jachowicz, K. Vantournhout, J. Ryckebusch, and K. Heyde, Phys. Rev. Lett. 93, 082501 (2004).

[52] E. Leader, Cambridge Monogr. Part. Phys Nucl. Phys. Cosmol. 15, 1 (2001).

[53] X. Artru, M. Elchikh, J.-M. Richard, J. Soffer, and O. V. Teryaev, Phys. Rep. 470, 1 (2009).

[54] V. Punjabi, C. F. Perdrisat, M. K. Jones, E. J. Brash, and C. E. Carlson, Eur. Phys. J. A 51, 79 (2015).

[55] M. Düren, The HERMES experiment: From the design to the first results, Ph.D. thesis, Erlangen-Nuremberg University, 1995.

[56] H. Middleton, G. Gates, T. Chupp, B. Driehuys, E. Hughes, J. Johnson, W. Meyer, N. Newbury, T. Smith, and A. Thompson, AIP Conf. Proc. 293, 244 (1993).

[57] J. Cao, A. de Gouvea, D. Duchesneau, S. Geer, R. Gomes, S. B. Kim, T. Kobayashi, K. R. Long, M. Maltoni, M. Mezzetto, N. Mondal, M. Shiozawa, J. Sobczyk, H. A. Tanaka, M. Wascko, and G. Zeller, arXiv:1704.08181.

[58] D. Brailsford, arXiv:1804.04979.
[59] C. Adams, R. An, J. Anthony, J. Asaadi, M. Auger, S. Balasubramanian, B. Baller, C. Barnes, G. Barr, M. Bass et al., J. Instrum. 13, P07007 (2018).

[60] See Supplemental Material at http://link.aps.org/ supplemental/10.1103/PhysRevD.101.073002 for analytic expressions for the spin asymmetry vector and tensors as well as spin asymmetry components.

[61] J. F. Donoghue, E. Golowich, and B. R. Holstein, Dynamics of the Standard Model, 2nd ed., Cambridge Monographs on Particle Physics, Nuclear Physics and Cosmology (Cambridge University Press, Cambridge, United Kingdom, 2014).

[62] W. M. Alberico, S.M. Bilenky, C. Giunti, and K. M. Graczyk, Phys. Rev. C 79, 065204 (2009).

[63] A. Thomas and W. Weise, The Structure of the Nucleon (Wiley, New York, 2010).

[64] C. Patrignani et al. (Particle Data Group), Chin. Phys. C 40, 100001 (2016).

[65] J. A. M. Vermaseren, arXiv:math-ph/0010025.

[66] R. Mertig, M. Bohm, and A. Denner, Comput. Phys. Commun. 64, 345 (1991).

[67] V. Shtabovenko, R. Mertig, and F. Orellana, Comput. Phys. Commun. 207, 432 (2016). 